\title{
Stimulus-Dependent Neuronal Oscillations and Local Synchronization in Striate Cortex of the Alert Cat
}

\author{
Charles M. Gray ${ }^{1}$ and Gonzalo Viana Di Prisco ${ }^{2}$ \\ 1 Section of Neurobiology, Physiology, and Behavior, The Center for Neuroscience, University of California, Davis, Davis, \\ California 95616, and ${ }^{2}$ Catedra de Fisiologia, Escuela de Medicina J. M. Vargas, Universidad Central de Venezuela, \\ Caracas 1010, Venezuela
}

Neuronal responses to visual stimuli that are correlated on a millisecond time scale are well documented in several areas of the mammalian visual cortex. This coherent activity often takes the form of synchronous rhythmic discharges ranging in frequency from 20 to $70 \mathrm{~Hz}$. We performed experiments to determine the incidence and properties of this rhythmic activity in the striate cortex of alert cats and to compare this activity to similar data collected in the striate cortex of anesthetized cats. The results demonstrate that optimal visual stimuli evoke robust, locally synchronous, $20-70 \mathrm{~Hz}$ oscillatory responses in the striate cortex of cats that are fully alert and performing a visual fixation task. The oscillatory activity is stimulus dependent, largely absent during periods of spontaneous activity, and shows a systematic increase in frequency with increasing stimulus velocity. Thus, the synchronous oscillatory activity observed in this and earlier studies cannot be explained as an artifact of anesthesia nor as a phenomenon that occurs independent of visual stimulation. Rather, it is a robust process that is present in the alert state and is dependent on the presence and specific properties of visual stimuli.

Key words: striate cortex; physiology; synchronization; oscillation; autocorrelation; gamma-band
In the visual cortex of cats and monkeys, a significant fraction of neurons in striate and prestriate areas exhibit pronounced rhythmicity in their firing patterns in response to optimal visual stimuli presented within their receptive fields (Gray and Singer, 1987, 1989; Eckhorn et al., 1988, 1993; Gray et al., 1990, 1995b; Engel et al., 1991b; Kreiter and Singer, 1992; Frien et al., 1994; Livingstone, 1996; see also Young et al., 1992; Bair et al., 1994). These stimulus-dependent neuronal oscillations occur mainly in the gamma frequency band $(20-70 \mathrm{~Hz})$, are largely absent during spontaneous firing, and are synchronized on a millisecond time scale (i.e., with time lags of $\pm 5 \mathrm{msec}$ ) over a range of spatial scales (Eckhorn et al., 1988; Gray et al., 1989; Engel et al., 1990, 1991a,b; Frien et al., 1994; Friedman-Hill et al., 1995; König et al., 1995a; Livingstone, 1996). Recent evidence indicates that this type of correlated firing between cells recorded in spatially separate columns occurs most often when one or both of the cells fire rhythmically in the gamma frequency band (Frien et al., 1994; König et al., 1995b; Friedman-Hill et al., 1995; Livingstone, 1996). This common finding suggests a functional link between gammaband oscillations and response synchronization.

Until recently (Frien et al., 1994; Friedman-Hill et al., 1995), the bulk of the evidence for gamma-band oscillations and response synchronization has come from studies in the anesthetized/paralyzed cat. This has raised the issue that these phenomena may reflect an artifact of anesthesia, or may have different properties in the alert animal. In an earlier report, Raether et al.

Received June 13, 1996; revised Jan. 31, 1997; accepted Feb. 5, 1997.

This work was supported by a grant from the National Science Foundation and fellowships from the Klingenstein Foundation and the Sloan Foundation. We thank Raul Aguilar and Dwight Corley for their invaluable computer software support and Lee Rognlie-Howes for her excellent care of the animals and technical assistance. Correspondence should be addressed to Dr. Gray at the above address.

Dr. Di Prisco's present address: Center for Complex Systems, Florida Atlantic University, Boca Raton, FL 33431.

Copyright (C) 1997 Society for Neuroscience $\quad 0270-6474 / 97 / 173239-15 \$ 05.00 / 0$
(1989) demonstrated synchronous oscillatory activity in area 17 of the alert cat. The properties of the activity, including frequency, phase and dependence on orientation, and ocular conditions of stimulation, were found to be similar to those observed in the anesthetized/paralyzed cat (Gray and Singer, 1989; Gray et al., 1990; Engel et al., 1990). The results, however, were taken from a very small number of recordings, and no provision was made to monitor or control the position of the eyes of the animal during visual stimulation. For these reasons, a systematic evaluation of the gamma-band activity in the alert animal was not possible, and the contribution of eye movements to the activity patterns could not be determined.

In the present study, we have recorded the activity of neurons in area 17 of three alert cats trained to fixate a central spot for up to $3 \mathrm{sec}$. We find oscillatory firing patterns present in a significant fraction of the recorded cells. The properties of this activity, including its frequency distribution and amplitude, are similar to those observed in the anesthetized/paralyzed cat, and contrary to an earlier report (Ghose and Freeman, 1992), we find that gamma-band activity is clearly stimulus dependent.

Parts of this paper have been published previously in abstract form (Gray and Viana Di Prisco, 1993).

\section{MATERIALS AND METHODS}

Surgery: alert cats. Three adult cats (two spayed females and one neutered male) were used in the present study. Before behavioral training, the animals were given surgical implants, using sterile technique, of a headpost for restraint, and a scleral search coil for eye position monitoring. The animals were given a preanesthetic cocktail of ketamine $(15 \mathrm{mg} / \mathrm{kg})$ and xylazine $(1 \mathrm{mg} / \mathrm{kg})$, and maintained under surgical anesthesia throughout the procedure using halothane (1-2\%) and oxygen. Body temperature was maintained at $38^{\circ} \mathrm{C}$, and the $\mathrm{EKG}$ and breathing rate were monitored continuously. The eye coil was implanted first using the technique of Judge et al. (1980). After this procedure, the animal was mounted sternally in a stereotaxic frame, and an incision was made along the midline over the skull. The periosteum was removed, and 6-10 
stainless steel skull screws $\left(4-40,3 / 16^{\prime \prime}\right)$ were inserted into the bone posterior to the frontal sinus. The head bolt was positioned over the skull screws using a manipulator mounted on the stereotaxic frame, and the assembly was covered with a thick layer of dental acrylic and allowed to harden. The two ends of the eye coil wire were fitted with crimp connectors (Amphenol) and mounted in a connector strip. This strip was embedded in dental acrylic adjacent to the head post. Both ends of the incision were closed with 4.0 nylon suture. The animals were given intraoperative fluids and antibiotics (keflin, $40 \mathrm{mg} / \mathrm{kg}$ i.v., once during the middle of surgery and once at the end of surgery), as well as a postoperative analgesic (buprenex, $0.05 \mathrm{mg} / \mathrm{kg}$ i.m., every $12 \mathrm{hr}$ for $36 \mathrm{hr}$ ).

The animals were allowed $10-14 \mathrm{~d}$ to recover from the effects of the surgery before beginning behavioral training. Once the training was complete (see below), a second sterile surgery was performed. A craniotomy $(5 \times 12 \mathrm{~mm})$ was made on one side overlying the representation of the area centralis of area 17. A recording chamber, fashioned out of hard plastic (Delrin), was implanted over the craniotomy and secured to the skull with 4-6 additional screws and dental acrylic. Induction and maintenance of anesthesia, as well as postoperative care, were identical to the first surgical procedure. The animals were again given 10-14 d to recover from the effects of surgery before electrophysiological recording was begun.

Surgery: anesthetized cats. Electrophysiological recordings were performed on 11 adult cats of both sexes using previously published techniques (Gray et al., 1990). On the day of the experiment, the animals were anesthetized with an i.m. injection of ketamine $(12 \mathrm{mg} / \mathrm{kg})$ and xylazine $(1 \mathrm{mg} / \mathrm{kg})$ and given atropine $(0.05 \mathrm{mg} / \mathrm{kg} \mathrm{s.c.})$ to reduce salivation. The cephalic vein was cannulated, and a continuous infusion of Ringers containing $2.5 \%$ dextrose was given throughout the experiment $(4 \mathrm{ml} /$ $\mathrm{kg} / \mathrm{hr})$. Anesthesia was maintained using halothane $(0.6 \%-1.5 \%)$ in a mixture of nitrous oxide and oxygen (2:1) while the animals were actively ventilated using a respirator pump. The EKG, heart rate, rectal body temperature, and expiratory $\mathrm{CO}_{2}$ were monitored continuously, the latter three being maintained within the ranges of $140-180 \mathrm{bps}, 37.5-39.0^{\circ} \mathrm{C}$, and $3.5-4.5 \%$, respectively. The animals were mounted in a stereotaxic frame, and a small craniotomy was made in one hemisphere over the representation of the area centralis of area 17. After the surgery, the animals were paralyzed with pancuronium bromide (Pavulon) using an initial bolus dose of $3 \mathrm{mg} / \mathrm{kg}$, followed by a continuous infusion of 3 $\mathrm{mg} / \mathrm{kg} / \mathrm{hr}$ (i.v.). The eyes were focused on the screen of a computer monitor at a distance of $57 \mathrm{~cm}$ using the tapetal reflection technique (Pettigrew et al., 1979) and an appropriate pair of gas permeable contact lenses. After these procedures, a small opening was made in the dura matter, a single electrode was positioned just above the cortical surface, and a $4 \%$ mixture of agar in Ringers solution was applied to the cortical surface to reduce pulsations. The assembly was then covered with molten bone wax.

Behavioral training. Behavioral training was carried out using pureed cat food as a reward (Prescription Diet). Because the training was best accomplished when the animals were hungry, it became necessary to assess the caloric requirements of each animal. Therefore, for a period of 3-6 weeks before any training or surgery, we determined the average daily caloric intake of each animal needed to maintain a stable daily body weight. After the animals reached a stable body weight, their daily food intake on Sunday through Thursday was reduced by $10-20 \%$. On Fridays and Saturdays they were given their full dietary allotment. The animals were inspected and weighed on a daily basis by ourselves and the veterinary staff. If at any stage during the training and recording experiments the weight of the animal dropped below $80 \%$ of the previous baseline, their full dietary complement was restored, and the training or recording was stopped.

Behavioral training was carried out in a three stage process. First, the naive animals were brought into the laboratory, placed in a cat bag for restraint, and given access to pureed cat food delivered from a small spout by an electric pump. This phase lasted about 1-3 weeks or until the animals became familiar with the restraint in the bag. The animals received all of their food in the laboratory. Once the animals became familiar with the restraint the first surgical procedure was performed.

The second phase consisted of training the animals to look at a small spot in the center of a computer screen placed at a distance of $57 \mathrm{~cm}$. The animals were placed in the cat bag, and the headpost was fixed to a frame. Initially, it was necessary to gradually familiarize the animals with this new form of restraint. On the first several days, head restraint lasted only a few minutes at a time. The duration of the restraint was gradually increased until the animals would remain calm and eat their food for
15-20 min. Anytime the animals vocalized or struggled, they were immediately removed from the restraint and placed in their home cage. This was important to ensure that the restraint was always associated with positive reinforcement. After the animals were thoroughly familiarized, a small spot of light was presented at roughly $10 \mathrm{sec}$ intervals while their eye position was tracked using the search coil. The animal was required to bring its gaze within a $5^{\circ}$ radius of the spot within a period of $2 \mathrm{sec}$ and maintain it there for at least $0.5 \mathrm{sec}$. If successful, the cat received a small bolus of the cat food from the spout placed directly in front of its mouth. Two types of incorrect trials occurred. Either the animals failed to bring their gaze within the fixation window within the required time or their gaze shifted outside the window during the fixation period. In each case, the trial ended and a brief delay period ensued.

As the animal approached an $80 \%$ success rate at this task, the difficulty of the task was gradually increased. The radius of the fixation window was reduced, and the time required to maintain its gaze within the window was increased. This process was continued until the two parameters were $1.0-1.5^{\circ}$ and $3 \mathrm{sec}$, respectively. The performance of the animals on this task varied widely, and it was difficult to tell at the outset which animals would be the quickest to learn. One animal learned the basic fixation task within 2 weeks, whereas another took up to 3 months.

The third phase of training consisted of teaching the animals to maintain their gaze within the fixation window during the presentation of 1-4 moving or stationary bars presented at varying locations and moving in varying directions and velocities. Initially, a small stationary bar of low luminance was presented in the periphery of the monitor. Over the course of days to weeks the bar was increased in size and luminance and presented at all locations on the monitor. The final step in this process was the introduction of multiple moving bars during the fixation trials. The animals gradually learned to maintain their gaze within the fixation window even when the bars moved across the fixation point. Once the animals were performing at approximately $80 \%$ correct, the second surgery was conducted to implant the recording chamber. Overall behavioral performance varied between animals, but we generally found that a given animal would work for 100-250 correct trials over a period of 30-120 $\mathrm{min}$.

Electrophysiological recording and visual stimulation. The techniques for unit recording and visual stimulation using anesthetized animals have been published previously (Gray et al., 1990; Maldonado and Gray, 1996) and were similar to those used with the alert animals. We therefore limit our description of the recording and stimulation methods to those used for the behavioral experiments.

Once the animals were fully trained to perform the fixation task, electrophysiological recordings were carried out on a daily basis. We used two slightly different techniques for the manipulation of our recording electrodes. For both techniques, the electrodes (1-2 M $\Omega$, tungsten) were advanced using miniature micromanipulators available from Biela Engineering (Malpeli et al., 1992). These manipulators, approximately $5 \mathrm{~mm}$ in diameter and $20-30 \mathrm{~mm}$ in length, were small enough to easily enable the placement of two independently movable microelectrodes within the recording chamber. We mounted the manipulators on a small Delrin insert, placed the insert within the recording chamber, and fixed it in place using set screws mounted in the walls of the chamber. Once in place, the electrodes were advanced through the dura into the cortex by manually turning the microdrives. The signals were bandpass filtered (600 $\mathrm{Hz}$ to $6 \mathrm{kHz})$, amplified $(5-10 \mathrm{k})$, fed through a Schmidt trigger, and digitized at a rate of $1 \mathrm{kHz}$.

After stable unit activity was isolated, the fixation task was initiated, and the receptive fields of the recorded cells were mapped using the minimum response field method (Barlow et al., 1967). This procedure typically required at least 20-30 trials to complete. Immediately after the mapping, a recording session was initiated in which the cells were stimulated by passing a light bar of optimal orientation, direction, and velocity over the receptive field. The stimulus was presented 10-20 times with an intertrial interval of $8-12 \mathrm{sec}$. In some sessions, the stimulus trials were pseudorandomly mixed with an equal number of trials containing no stimulus to permit an evaluation of the properties of the spontaneous activity. After this sequence was complete, the electrode was advanced to a new position, and the procedure repeated if the behavioral performance of the animal permitted. At the end of each session, the electrodes were retracted, the insert containing the manipulators removed, and the chamber cleaned and sealed with a cap.

After conducting many sessions using these recording techniques, it became apparent that the method suffered from a long setup time. The insert had to be carefully placed, the electrodes advanced into the tissue, 
stable unit recordings obtained, and the receptive fields mapped before data could be collected. This sequence often required $30-40 \mathrm{~min}$ and $30-40$ behavioral trials, thereby reducing the available time for collecting data. In an attempt to improve the yield, we resorted to leaving the electrodes in place at the end of an initial recording session. This proved to be a rather simple procedure, and the manipulators were protected by a hard cap made out of Delrin that we fixed to the recording chamber with screws. On subsequent days, the setup procedure was now reduced to 5-10 min. The protective cap was removed and the electrode connector plugged in. A quick check could be made to see whether unit activity was still present at the recording site, and if not, the manipulator was advanced slowly until new units were isolated. Because of the columnar and retinotopic organization of the striate cortex, the time needed to map the receptive fields of the new cells was dramatically reduced. Overall, this technique greatly improved the yield of the experiments and seems to be essential when using cats as subjects because of the limited time available to record.

In addition to the simple sequences of optimal bar stimulation, we also investigated the influence of changes in stimulus velocity on the properties of oscillatory responses. In a subset of sessions, we performed velocity tuning curves using 3-6 different velocities ranging from $0.9^{\circ} / \mathrm{sec}$ to $12 \%$ sec presented in a pseudorandom order. The range of velocities chosen was centered around the preferred velocity for any given recording. All stimuli ranged in luminance from 0.1 to $20 \mathrm{~cd} / \mathrm{m}^{2}$ and were presented on a dark background.

Data analysis. The amplitude and time-dependent properties of the recorded spike trains were evaluated using several quantitative measures. First, we computed a peristimulus time histogram (PSTH) for each recording using a bin width of $50 \mathrm{msec}$. The mean firing rate was computed from each recording during two epochs, one before stimulus presentation during spontaneous activity, and the second during the period of stimulus presentation. We refer to these two epochs as window 1 (win1) and window 2 (win2), respectively. The duration of these windows ranged from $0.6-2$ sec. Tuning curves were constructed by computing both the mean and peak firing rate within each of the two windows and plotting the results as a function of stimulus number.

To evaluate the time-dependent properties of the spike trains, we computed the autocorrelation histogram $(\mathrm{ACH})$ on the activity present within both time windows. This was done for individual trials, and the sum of the activity recorded across all trials. As a control for periodicities in the spike trains introduced by the computer monitor $(80 \mathrm{~Hz}$ noninterlaced refresh rate), we also computed the cumulative autocorrelation histogram across trials after shuffling the trial sequence by one stimulus period (i.e., the shift predictor). Any stimulus-locked frequencies present in the spike train would be present in the shift predictor correlogram. We used an additional measure to compare the resulting correlograms with those that are computed from an equivalent random process. For the data collected on each trial within each of the two windows, we generated a spike train having a pseudorandom distribution of intervals with an equivalent number of spikes at the same mean firing rate. These data sets, serving as a control, were subjected to autocorrelation analysis in exactly the same manner as the experimental data. From the resulting correlation histograms, we computed the mean and SD across all bins and used these values to estimate the confidence limits for significant peaks and/or troughs in the experimental correlograms. For all the correlogram calculations, we used positive and negative time lags of both $128 \mathrm{msec}$ and 256 msec at a bin width of $1 \mathrm{msec}$.

To evaluate the temporal structure of each ACH (single trial, across trials, shift predictor, random control, win 1 and win2), we computed the power spectrum of the ACH using the fast fourier transform (Press et al., 1992). From each spectrum obtained from the experimental data, we extracted three measures to quantify their properties. First, a peak detection routine was applied to determine the magnitude and frequency of the peak value in the spectrum from $20-70 \mathrm{~Hz}$, as well as the magnitude of the DC component. The frequency of the peak value and the ratio of the peak value to the DC component (peak/DC) provide the first two measures of the spectra. These values were considered significant if the peak between $20-70 \mathrm{~Hz}$ exceeded the mean plus three SDs of the corresponding control power spectrum (excluding the DC component) computed from the ACH of the pseudorandom spike train. As a third measure, we computed the signal to noise ratio $(\mathrm{S} / \mathrm{N})$ of the power spectra (Ghose and Freeman, 1992). The signal was computed by taking the average over an $8 \mathrm{~Hz}$ window centered on the peak in the spectrum between $20-70 \mathrm{~Hz}$. The noise for each spectrum was computed by taking the average of all the values between 250 and $500 \mathrm{~Hz}$.
For each of these three measures, we examined the cumulative distributions for correlation histograms computed across trials and on individual trials. Because single trials often did not yield a sufficient number of spikes to reliably employ autocorrelation analysis, we devised a simple criterion for the number of spikes needed, below which the correlograms were excluded from the analysis. For each correlogram (across trials and individual trials), the mean of the corresponding pseudorandom control correlogram had to be equal to or greater than twice its SD. Although this criterion was arbitrary, it served to provide a discrete cutoff for acceptance that was directly related to the criterion for statistical significance.

\section{RESULTS}

\section{Incidence, magnitude, and stimulus dependence of oscillatory activity}

The main focus of this report is the analysis of oscillatory activity in the striate cortex of the alert cat. Because the properties of this activity in the anesthetized cat have been documented extensively (Gray and Singer, 1989; Gray et al., 1989, 1990; Engel et al., 1990), the data collected from anesthetized animals in this study are used primarily for the purpose of comparison. Our aim in this regard is to compare the incidence of occurrence, the magnitude, and the frequency distribution of oscillatory activity under the two conditions. Our sample consisted of 128 multiunit and 17 single unit recordings from 3 alert cats, and 100 multiunit and 48 single unit recordings from 11 anesthetized cats.

An example of multiunit activity recorded from an alert cat during the presentation of an optimally oriented, drifting light bar is shown in Figure 1. The animal maintained fixation for $2.4 \mathrm{sec}$ and the stimulus was presented at a latency of $0.6 \mathrm{sec}$ after the start of fixation. Initially, the light bar appeared outside the receptive field and then passed through it. Therefore, the peak of the response occurred roughly between 1 and $2 \mathrm{sec}$ into the trial. The timing of the behavioral task, shown in the middle portion of the figure, allowed us to select two epochs of data for further analysis. These epochs, termed win1 and win2, correspond to periods of spontaneous and stimulus-evoked activity. The quantitative analysis of these data are shown in Figure 2. The ACH was computed across all 10 trials in the session for win1 (Fig. 2A), win2 (Fig. 2C), and the shift predictor control of win2 (Fig. 2E). The horizontal lines in Figure 2, $A, C$ and $E$, represent the mean and the mean \pm 2 SDs (i.e., 95\% confidence limits) of the autocorrelation histogram computed from an equivalent pseudorandom spike train. In all 3 histograms, the mean is greater than twice the $\mathrm{SD}$, although in Figure $2 A$ by only a slight margin. Thus, each of these data sets met our criteria for sufficient numbers of spikes. Any data set not meeting this criteria was excluded from the cumulative results. The power spectra of the corresponding ACHs are shown in Figure 2, $B, D$, and $F$. The thick and thin lines in these graphs represent the mean and the mean +3 SDs $(99 \%$ confidence limit) of the power spectra computed from the equivalent pseudorandom spike trains. These data illustrate several points; statistically significant rhythmic firing is present during the response to the stimulus and absent during spontaneous firing. The absence of any peaks in the shift predictor control demonstrates that the oscillations are not time-locked to the stimulus. This latter result excludes the possibility that the rhythmic firing is driven by the video refresh $(80 \mathrm{~Hz})$ of the computer monitor.

In the same recording, we found that oscillatory activity was easily detectable on single trials. Figure 3 shows three trials of activity, recorded during a separate trial block, at the same site as the data shown in Figures 1 and 2. The spike trains, along with the vertical and horizontal eye position, are shown in Figure $3 A$. The receptive field of the cells was located at an eccentricity of $1.8^{\circ}$, 


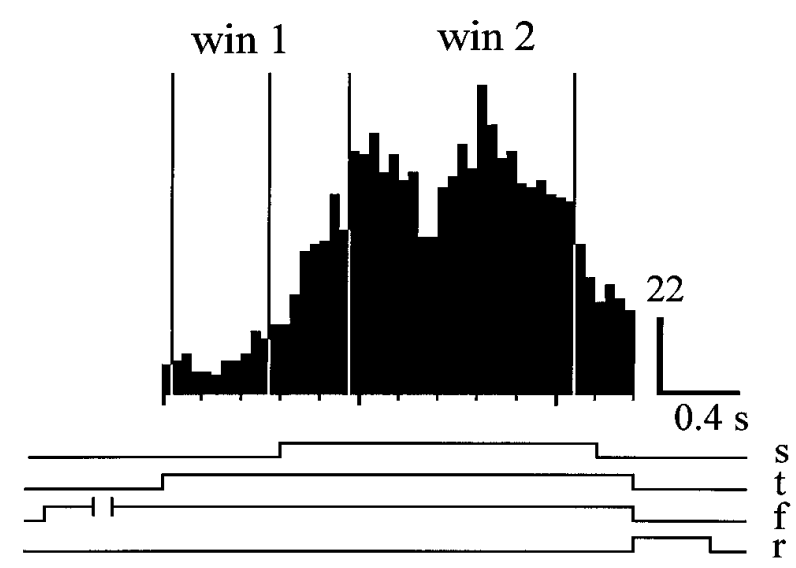

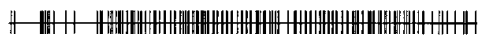

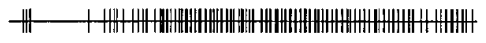

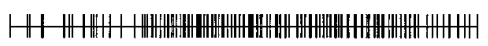

十\#-

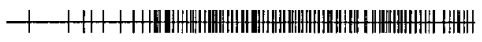

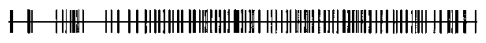

†

†

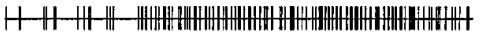

H

Figure 1. The behavioral paradigm used for recording unit activity from an alert cat trained to visually fixate a central target. The middle four traces schematically illustrate the temporal sequence of events that occur during a single trial. $s, t, f$, and $r$ represent the time of occurrence of the stimulus, the period of data acquisition, the fixation spot, and the food reward, respectively. The top plot is a PSTH of multiunit activity recorded across 10 consecutive trials. win 1 and win2 illustrate the epochs selected for correlation analysis and reflect periods of spontaneous and stimulusevoked activity, respectively. The bottom traces are raster plots of the 10 spike trains.

and the bar passed over the center of gaze while moving across the receptive field. Thus, even though small saccades and slow drifts in eye position of roughly $0.5^{\circ}$ are evident, the animal was able to supress a smooth pursuit eye movement during the stimulus presentation. The ACHs and associated power spectra computed for each trial during win2 are shown in Figure $3, B$ and $C$, respectively. Strong oscillatory activity near $40 \mathrm{~Hz}$ is present on each trial. This indicates that oscillatory firing among local groups of cells is highly synchronous and can be analyzed on a trial by trial basis from short epochs of data.

To quantify the properties of the rhythmic firing, we computed the peak/DC value, the frequency at the peak, and the $\mathrm{S} / \mathrm{N}$ (see Materials and Methods). These values are shown to the right of each spectrum in Figures 2 and 3, respectively. The peak/DC value provides a measure of the modulation amplitude of the $\mathrm{ACH}$ that is normalized for the number of spikes. The $\mathrm{S} / \mathrm{N}$ provides a measure of the modulation amplitude relative to the average of the high frequency $(250-500 \mathrm{~Hz})$ signals in the correlation histogram. To evaluate the cumulative properties of the data, we first sought to establish a set of criteria for statistical significance. For the peak/DC values, we used three criteria. First, for each trial and trial block, the mean value in the $\mathrm{ACH}$ of the equivalent pseudorandom spike train had to be equal to or greater
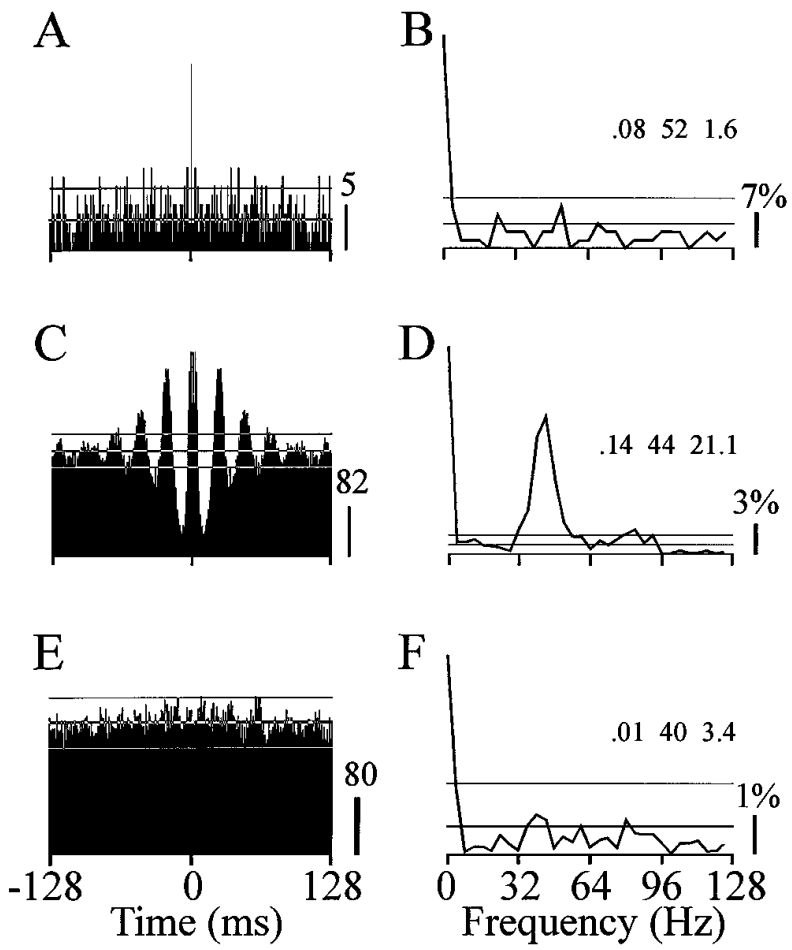

Figure 2. Autocorrelation histograms $(A, C, E)$ and corresponding power spectra $(B, D, F)$ computed from the multiunit recording shown in Figure 1 during periods of spontaneous $(A, B)$ and stimulus-evoked $(C, D)$ activity. $E$ and $F$ were computed from the data collected during win 2 after shuffling the spike trains by one stimulus period. Note the complete absence of significant peaks in the histogram in $E$. This indicates that the oscillatory activity is not phase-locked to the stimulus. The three horizontal lines passing through the autocorrelation histograms represent the mean and $95 \%$ confidence limits computed from the trial shuffled control (i.e., shift predictor). The thick and thin lines shown in the power spectra represent the mean and $99 \%$ confidence limit, respectively, for an equivalent random spike train. In this and subsequent figures, the three values shown to the right of each spectrum are the peak/DC value, the frequency of the peak, and the $\mathrm{S} / \mathrm{N}$, respectively.

than twice the SD. Second, the peak in the power spectrum had to exceed the mean +3 SDs of the power spectrum computed from the pseudorandom control. Third, the peak/DC value had to exceed the value at which $90 \%$ of all the peak/DC values, computed from the trial-shuffled correlograms, fell below. For the cumulative autocorrelation spectra recorded during visual stimulation this value was 0.06 for the alert and anesthetized data. During spontaneous activity (win1) this cutoff value was 0.10 and 0.09 for the alert and anesthetized data, respectively. According to these criteria, the stimulus-evoked oscillatory activity shown in Figure 2, $C$ and $D$, was statistically significant, whereas the data collected during spontaneous firing (Fig. $2 A, B$ ) or the shift predictor control (Fig. $2 E, F$ ) were not. In Figure 3, all three trials exhibited significant oscillatory firing during the stimulus presentation. For the $\mathrm{S} / \mathrm{N}$ values, we used the cutoff value of 1.5 as proposed previously by Ghose and Freeman (1992). Thus, spectra having an $\mathrm{S} / \mathrm{N}$ value greater than 1.5 were considered significant. In contrast to the peak/DC measure, the $\mathrm{S} / \mathrm{N}$ measure indicated that significant oscillatory firing was present in all three conditions of Figure 2 and for all three trials of Figure 3.

We applied these calculations to the cumulative ACHs for both the alert and anesthetized data sets. The results for the peak/DC and the peak frequency values computed during win2 are shown 

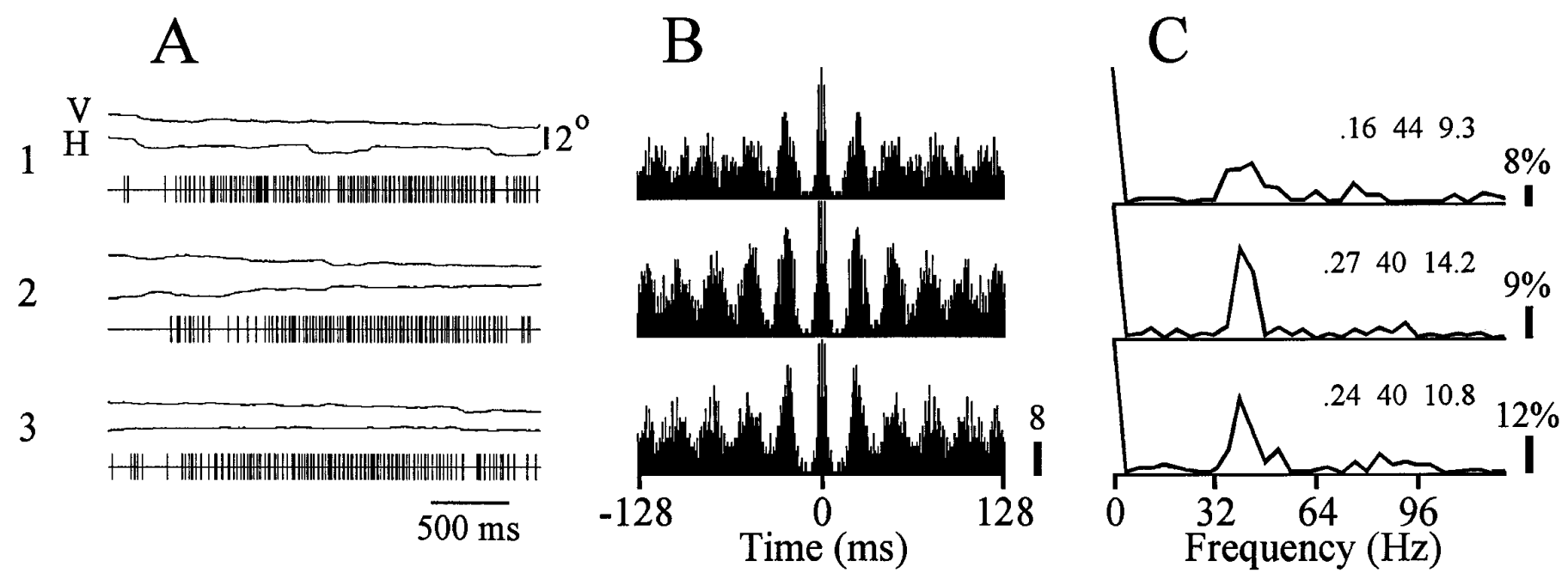

Figure 3. Stimulus-dependent oscillatory responses are clearly evident on single trials. Three trials (1-3) of the multiunit activity recorded in area 17 of an alert cat while the animal maintained fixation of a central spot. The data are taken from the same recording shown in Figures 1 and 2. A, Plots of vertical $(V)$ and horizontal $(H)$ eye position along with the spike train for each of the three trials. Vertical bar to the right is the calibration for eye position. $B$, Single trial autocorrelation histograms computed from the spike trains during the period of stimulus presentation. $C$, Power spectra of the corresponding autocorrelation histograms shown in $B$.
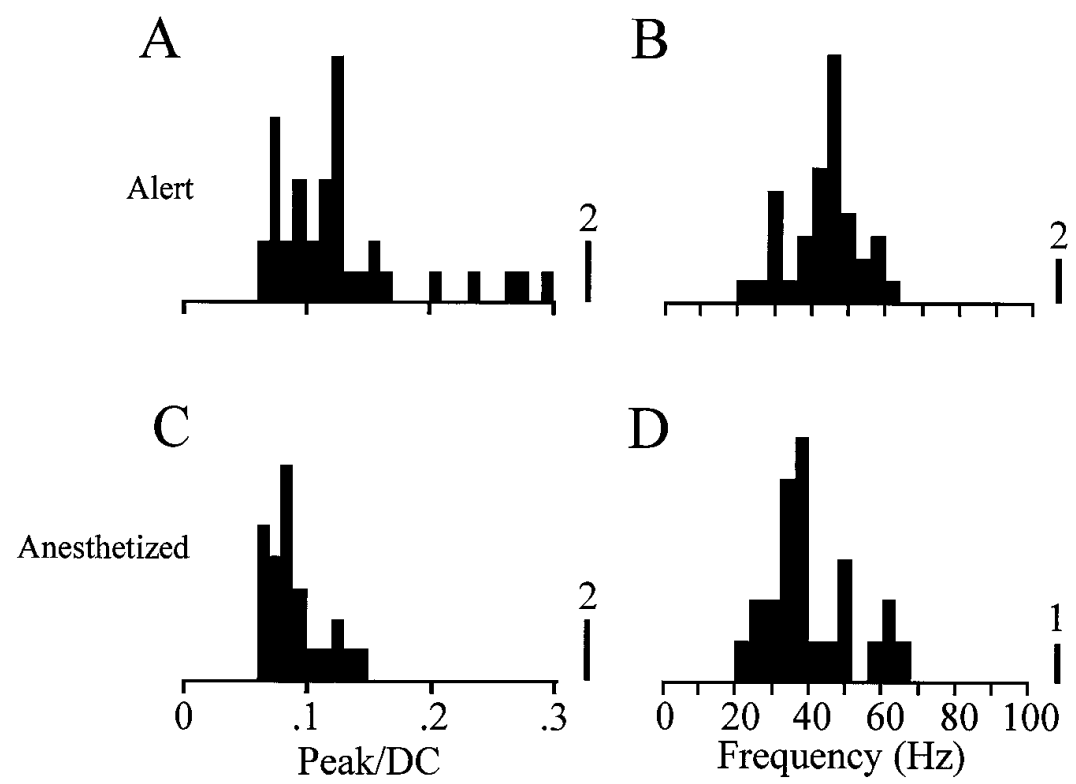

Figure 4. Cumulative histograms of the significant peak/DC values $(A, C)$ and the corresponding peak frequencies $(B, D)$ obtained from the power spectra of the autocorrelation histograms for the alert $(A, B)$ and anesthetized $(C, D)$ experiments, respectively.

in Figure 4 and Table 1 . The $\mathrm{S} / \mathrm{N}$ values for win2 are shown in Figure 5. Using the criteria described above for the peak/DC values, we found that 38 of $144(26.4 \%)$ recordings in alert animals and 25 of $148(16.9 \%)$ recordings in anesthetized animals showed significant oscillations. Analysis of the distributions in Figure 4, $A$ and $C$, revealed that the magnitude of the oscillatory activity was greater in the alert $(0.127 \pm-.057)$ than in the anesthetized $(0.089 \pm 0.022)$ state $(p<0.001$, Mann-Whitney $U$ test). Although this difference is significant, it is clear from Table 1 that differences in the sample size from each animal as well as individual variation in the incidence and magnitude of oscillatory activity could account for this result. The mean frequencies in the alert and anesthetized states were $41.4 \pm 9.4 \mathrm{~Hz}$ and $39.7 \pm 11.8$ $\mathrm{Hz}$, respectively. Neither the mean values nor the variance in peak frequency differed between the anesthetized and alert conditions.

In contrast to these results, we found that the criterion value of
1.5 for the $\mathrm{S} / \mathrm{N}$ measure was much more sensitive than the peak/DC measure (Fig. 5). This analysis detected significant oscillations in 122 of $144(85 \%)$ and 120 of $148(81 \%)$ recordings in the alert and anesthetized animals, respectively. We suspected that this high percentage of significant values might result from the fact that our autocorrelation calculations used a time lag of $\pm 128 \mathrm{msec}$. We therefore recomputed the $\mathrm{S} / \mathrm{N}$ values using a correlation time lag of $\pm 256 \mathrm{msec}$ (Ghose and Freeman,1992). With this calculation, we found that 104 of 144 (72\%) recordings showed significant oscillations in the alert state. The greater sensitivity of the $\mathrm{S} / \mathrm{N}$ compared to the peak/DC calculations seems to be a result of the arbitrary selection of 1.5 as a criterion for statistical significance. We examined the distribution of $\mathrm{S} / \mathrm{N}$ values for both data sets obtained from those recordings that met the criteria for significant peak/DC values and found that many of the spectra having low $(<8)$, as well as high $(>8), \mathrm{S} / \mathrm{N}$ values did 


\begin{tabular}{|c|c|c|c|}
\hline & No. of cells & Mean peak/DC & Mean frequency $(\mathrm{Hz})$ \\
\hline \multicolumn{4}{|c|}{ Behavioral } \\
\hline Cat 1 & 2 of $17(11.7 \%)$ & $0.095 \pm 0.03$ & $36 \pm 0$ \\
\hline Cat 2 & 4 of $44(9.1 \%)$ & $0.0829 \pm 0.0124$ & $47 \pm 6.8$ \\
\hline Cat 3 & 32 of $83(38.5 \%)$ & $0.1345 \pm 0.0592$ & $41 \pm 9.8$ \\
\hline Total & 38 of $144(26.4 \%)$ & $0.1270 \pm 0.0573$ & $41.4 \pm 9.4$ \\
\hline \multicolumn{4}{|c|}{ Anesthetized } \\
\hline Cat 1 & 1 of $15(6.7 \%)$ & 0.0958 & 32 \\
\hline Cat 2 & 1 of $7(14.3 \%)$ & 0.0783 & 28 \\
\hline Cat 3 & 3 of $10(30 \%)$ & $0.0775 \pm 0.01$ & $40 \pm 13.8$ \\
\hline Cat 4 & 7 of $11(63.6 \%)$ & $0.0905 \pm 0.0235$ & $39.4 \pm 5.8$ \\
\hline Cat 5 & 3 of $12(25 \%)$ & $0.0882 \pm 0.0362$ & $42.7 \pm 18.9$ \\
\hline Cat 6 & 0 of $15(0 \%)$ & & \\
\hline Cat 7 & 5 of $25(20 \%)$ & $0.0942 \pm 0.0189$ & $47.2 \pm 13.7$ \\
\hline Cat 8 & 2 of $15(13.3 \%)$ & $0.0836 \pm 0.0142$ & $28 \pm 4$ \\
\hline Cat 9 & 0 of $6(0 \%)$ & & \\
\hline Cat 10 & 1 of $20(5 \%)$ & 0.0894 & 32 \\
\hline Cat 11 & 2 of $12(16.7 \%)$ & $0.1168 \pm 0.0412$ & $32 \pm 16.9$ \\
\hline Total & 25 of $148(16.9 \%)$ & $0.0896 \pm 0.0218$ & $39.7 \pm 11.8$ \\
\hline
\end{tabular}

not meet the criteria applied to the peak/DC values. This indicates that many spectra having $\mathrm{S} / \mathrm{N}$ values $>1.5$ contained peaks that were no greater than those that would be expected to occur by chance. Thus, the $\mathrm{S} / \mathrm{N}$ measure is subject to a large percentage of false positive detections of oscillatory activity.

After applying a similar analysis to the data collected during spontaneous firing (win1), we found that the incidence of significant oscillations was much lower than that recorded in response to visual stimulation. This was, in large part, a result of the low spontaneous firing rate of most cells in striate cortex. In the alert and anesthetized states, respectively, 4 of 57 and 4 of 28 recordings, meeting the criteria for sufficient numbers of spikes, displayed significant oscillations. The frequency of this activity was slightly lower than that observed during visual stimulation (alert $=$ $30 \pm 9.5 \mathrm{~Hz}$; anesthetized $=29 \pm 2 \mathrm{~Hz}$ anesthetized).

We also applied the peak/DC analysis to the correlation histograms computed on individual trials. The results of these calculations are shown in Figure 6A,C. In the alert animals, 977 of 2115 trials met the criteria for sufficient numbers of spikes, whereas in the anesthetized animals, this value was 861 of 1975 total trials. Of these, significant peak/DC values were detected on 393 trials in the alert animals $(40.2 \%$; mean $=0.144 \pm 0.081)$ and 245 trials in the anesthetized animals $(28.5 \%$; mean $=0.117 \pm 0.045)$. As with the cumulative correlograms, the magnitude of the oscillatory activity was greater in the alert state $(p \ll 0.0001$, MannWhitney $U$ test). The distribution of peak frequencies (Fig. 6B,D) revealed that oscillation frequency was similar for the alert $[41.4 \pm 10.1 \mathrm{~Hz}(n=393)]$ and anesthetized $[40.9 \pm 12.3 \mathrm{~Hz}(n=$ $245)]$ states, although the frequency variance was greater in the data obtained from the anesthetized animals ( $p<0.0006, F$ test). During spontaneous firing, only 10 of 57 trials (alert data) and 4 of 20 trials (anesthetized data) having sufficient numbers of spikes displayed significant oscillations. Again, these low numbers stemmed largely from the very low firing rates of cells during spontaneous activity.

Because the oscillation frequency varies within as well as across trials (Gray et al., 1990, 1992), and this variance would be expected to average out the oscillatory components in the cumulative autocorrelograms, we suspected that our measure of the incidence of oscillatory activity (Fig. 4) underestimated the number of recordings that displayed oscillatory firing. To test this conjecture, we determined the percentage of trials at each recording site that displayed significant oscillatory modulation. The results of these calculations, shown in Figure 7, revealed that greater than $50 \%$ of the cells, in both the alert and anesthetized animals, exhibited significant oscillatory responses to visual stimuli on at least $10 \%$ of the trials. This finding has several implications. First, it indicates that the cumulative $\mathrm{ACH}$ underestimates the incidence of oscillatory activity. Second, it demonstrates that neuronal oscillations are episodic, nonstationary events. They may vary in frequency or in probability of occurrence or both. Thus, the failure to find oscillatory modulation in a cumulative $\mathrm{ACH}$ may result from too few trials displaying oscillations, or from a variation in oscillation frequency that smoothes the cumulative histogram. Examples of both of these effects recorded in the alert animals are shown in Figures 8 and 9, respectively. Figure 8 shows an example where the cumulative $\mathrm{ACH}$ (Fig. $8 A, B$ ), but none of the 20 individual trial histograms (Fig. $8 C, D$ ), met the criteria for significant oscillations. In those trials where it is possible to see oscillatory modulation (i.e., $3,7,10,11,13$, and 16), the
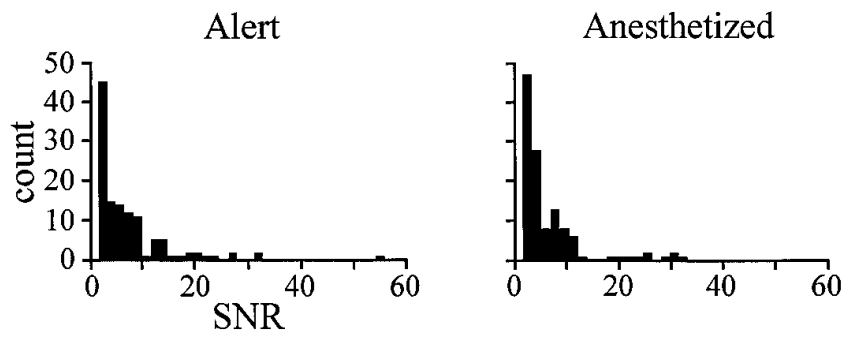

Figure 5. Cumulative histograms of the signal-to-noise ratios computed from the power spectra of the autocorrelation histograms during the response to stimuli for the alert and anesthetized experiments. Both plots show the distribution of $\mathrm{S} / \mathrm{N}$ values that exceed the two $\sigma$ criteria for sufficient spike counts and are $\geq 1.5$. 

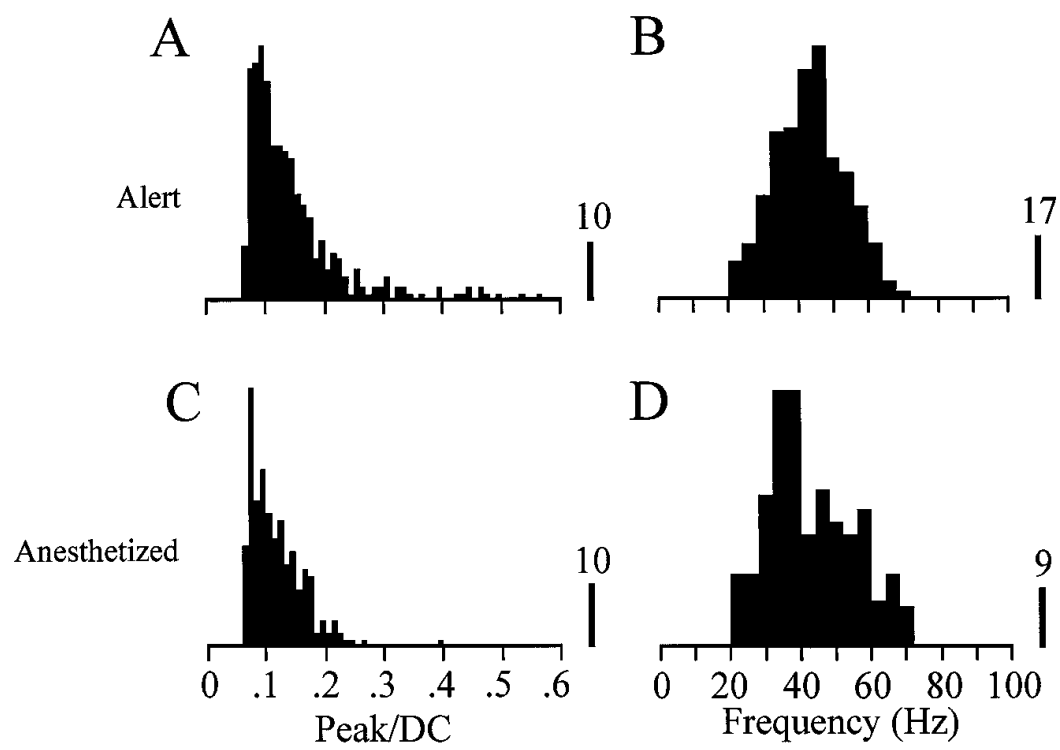

Figure 6. Cumulative histograms of the significant peak/DC
values $(A, C)$ and the peak frequencies $(B, D)$ obtained from
the power spectra of the autocorrelation histograms com-
puted on single trials during the presentation of visual stimuli
for the alert $(A, B)$ and anesthetized $(C, D)$ experiments,
respectively.

number of spikes was insufficient to meet the criterion. The remaining trials have either too few spikes or no rhythmic modulation. Despite these sources of variance, the cumulative ACH was highly significant. This is because the oscillatory activity, when it did occur, was relatively constant in frequency. Thus, summing the individual trials did not lead to an averaging out of the rhythmic modulation. Figure 9 shows a different example in which 8 of 10 single trial histograms (Fig. 9C,D), but not the cumulative $\mathrm{ACH}$ (Fig. $9 A, B$ ), met the criteria for statistical significance. Here it is clear that the variation of oscillation frequency accounts for the low modulation amplitude of the cumulative ACH. An example of this can be clearly seen by comparing trials 4 and 8 in Figure $9 C, D$. Thus, fre-

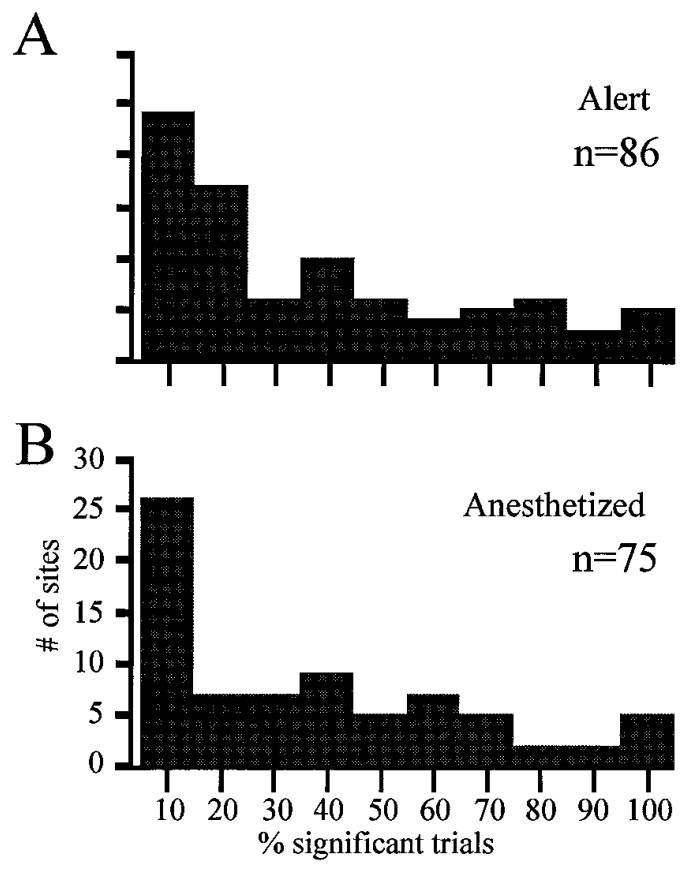

Figure 7. Frequency histograms of the percentage of trials recorded at each site that contained at least $10 \%$ of trials displaying significant, stimulus-evoked oscillations. $A$, Awake behaving condition. $B$, Anesthetized condition. quency variance, in addition to probability of occurrence, can account for the failure to observe significant oscillations.

Our effort to evaluate the occurrence of oscillatory firing during spontaneous activity was limited by the low firing rates in the absence of visual stimulation and, hence, a failure to collect sufficient numbers of spikes at many of the recording sites. We therefore changed our strategy during the experiments and ran sessions in which we interleaved stimulus trials with trials containing only a fixation spot, or in which we varied the stimulus luminance. This allowed us to sample spontaneous activity for longer periods of time and to compare its temporal properties to that evoked during visual stimulation. Figure 10 shows one example in which oscillatory activity evoked during visual stimulation (Fig. $10 B, D, F$ ) is absent during a comparable period of spontaneous firing while the animal is fixating (Fig. $10 A, C, E$ ). Similarly, Figure 11 illustrates a recording in which significant oscillations were absent at low levels of stimulus luminance but became highly significant as the response magnitude approached saturation. These examples were consistent with our sample of 14 multiunit recordings that displayed significant oscillatory responses to an optimal visual stimulus (mean $=41 \mathrm{~Hz}$ ), whereas in the absence of visual stimulation these cells showed significant oscillatory modulation in only 2 of the 14 cases $($ mean $=34 \mathrm{~Hz})$. Thus, in the absence of visual stimulation, rhythmic firing occurs less often and at a lower frequency among cells that display pronounced oscillations during visual stimulation.

We obtained further evidence for the stimulus dependence of oscillatory activity by comparing the frequency of oscillations at different velocities of stimulus movement. It has been shown previously in anesthetized animals (Eckhorn et al., 1988; Gray et al., 1990) that oscillation frequency increases with stimulus velocity. Our results in the alert cats were consistent with these earlier reports. In 11 of 12 multiunit recordings, we found that the frequency of significant oscillations evoked by a moving light bar increased monotonically with the velocity of stimulus movement. An example of this result, from a multiunit recording in area 18, is shown in Figure 12. A scatter plot of the cumulative data is shown in Figure 13. In this sample, significant oscillations spanned a frequency range of $36-60 \mathrm{~Hz}$ and occurred at stimulus velocities ranging from 1.4 to $11.8^{\circ} / \mathrm{sec}$. Linear regression analysis revealed a significant correlation between stimulus velocity and oscillation 

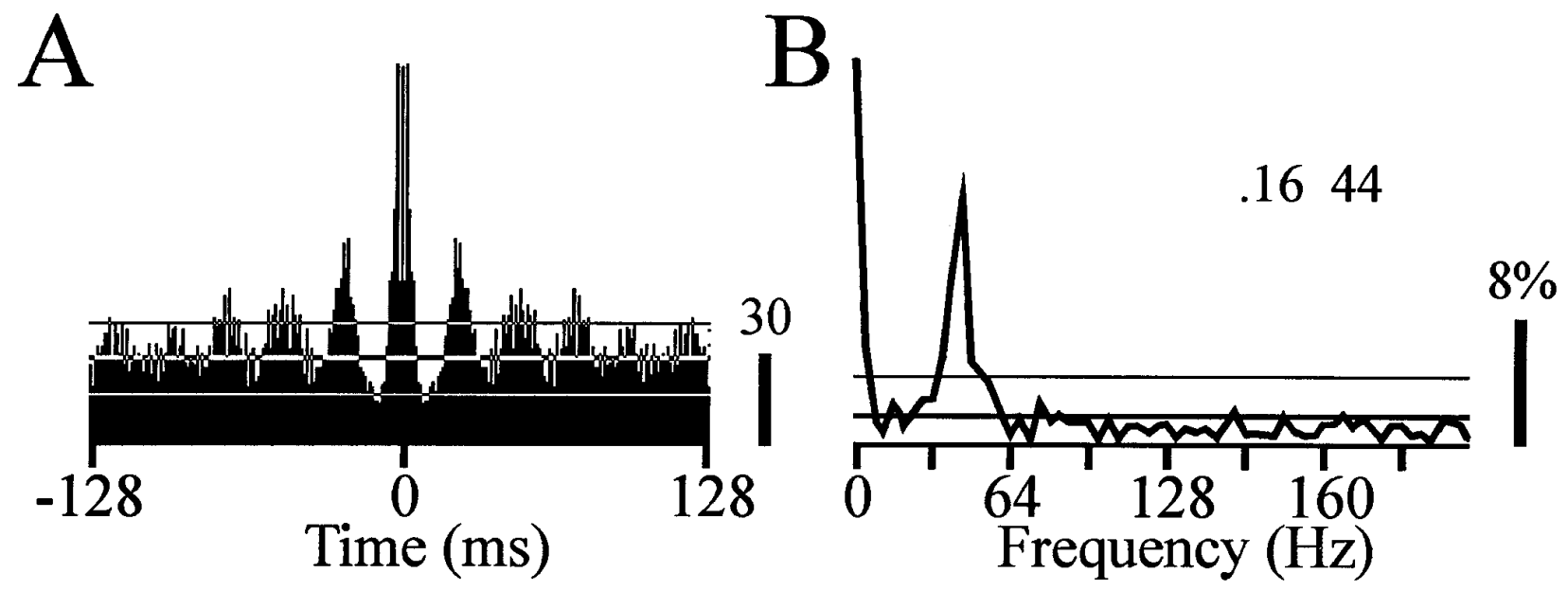

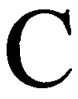

D
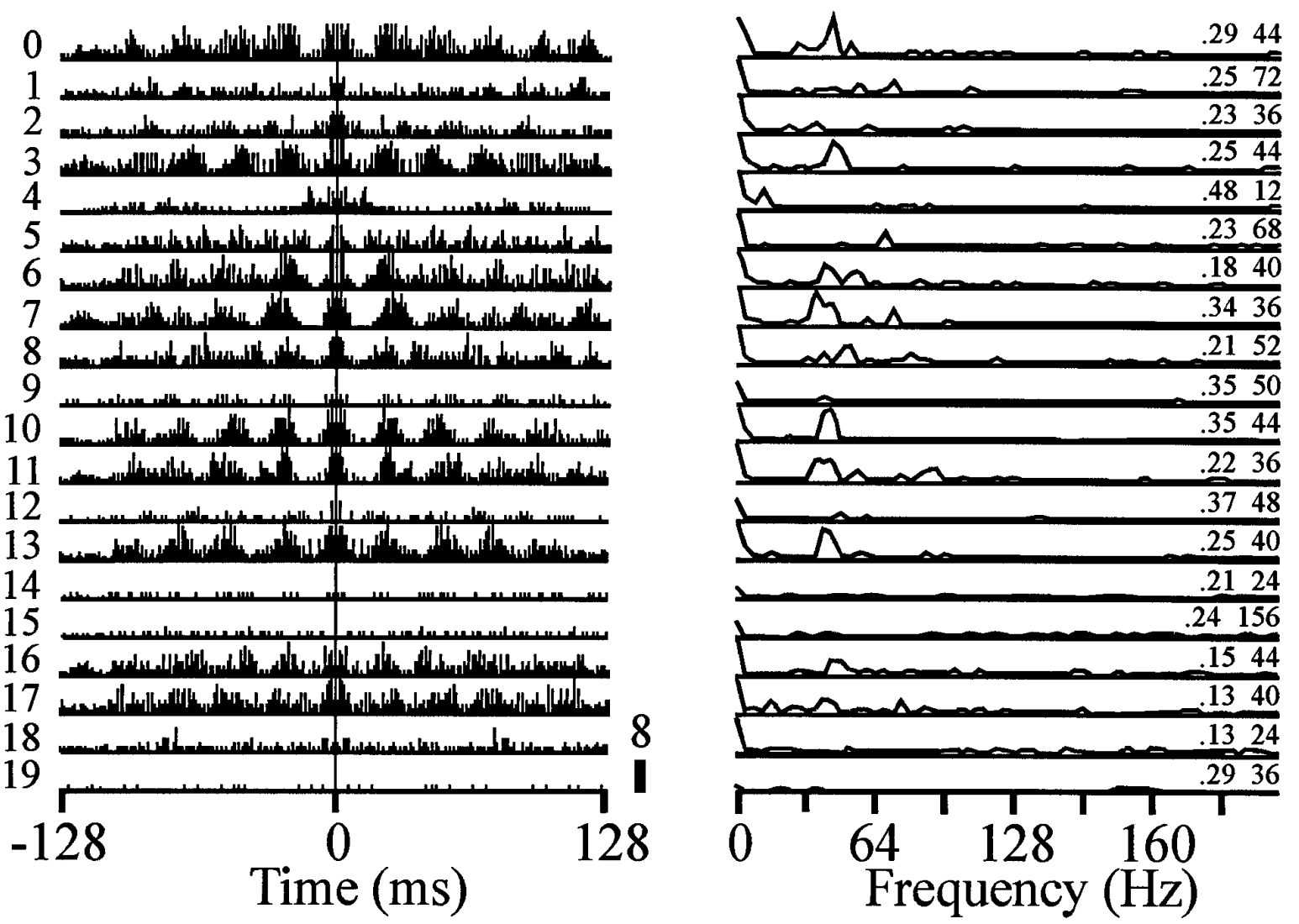

Figure 8. Example of a multiunit recording in which the cumulative autocorrelation histogram exhibits significant periodicity whereas none of the individual trials meet the criteria for significance. $A$, Cumulative autocorrelation histogram. The thick and thin horizontal lines represent the mean and 95\% confidence limits computed from the shift predictor, respectively. $B$, Power spectrum of the autocorrelation histogram shown in $A$. The autocorrelation histograms and associated power spectra for each of the 20 trials recorded on this session are shown in $C$ and $D$, respectively. None of the 20 trials met the criteria for statistical significance. They either lacked sufficient numbers of spikes or did not display significant power at any frequency.

frequency $(r=0.71, p \ll 0.0001)$ with frequency increasing by 1.5 $\mathrm{Hz}$ for each $1 \%$ sec increase in stimulus velocity. These results closely resemble those obtained in the anesthetized cat (Gray et al., 1990) and further demonstrate that the properties of oscillatory responses in cat striate cortex are stimulus dependent.

\section{Local synchronization}

In a subset of our multiunit recordings that displayed significant oscillations, we utilized spike sorting techniques (Abeles and Goldstein, 1977; Gray et al., 1995a) to enable us to analyze 


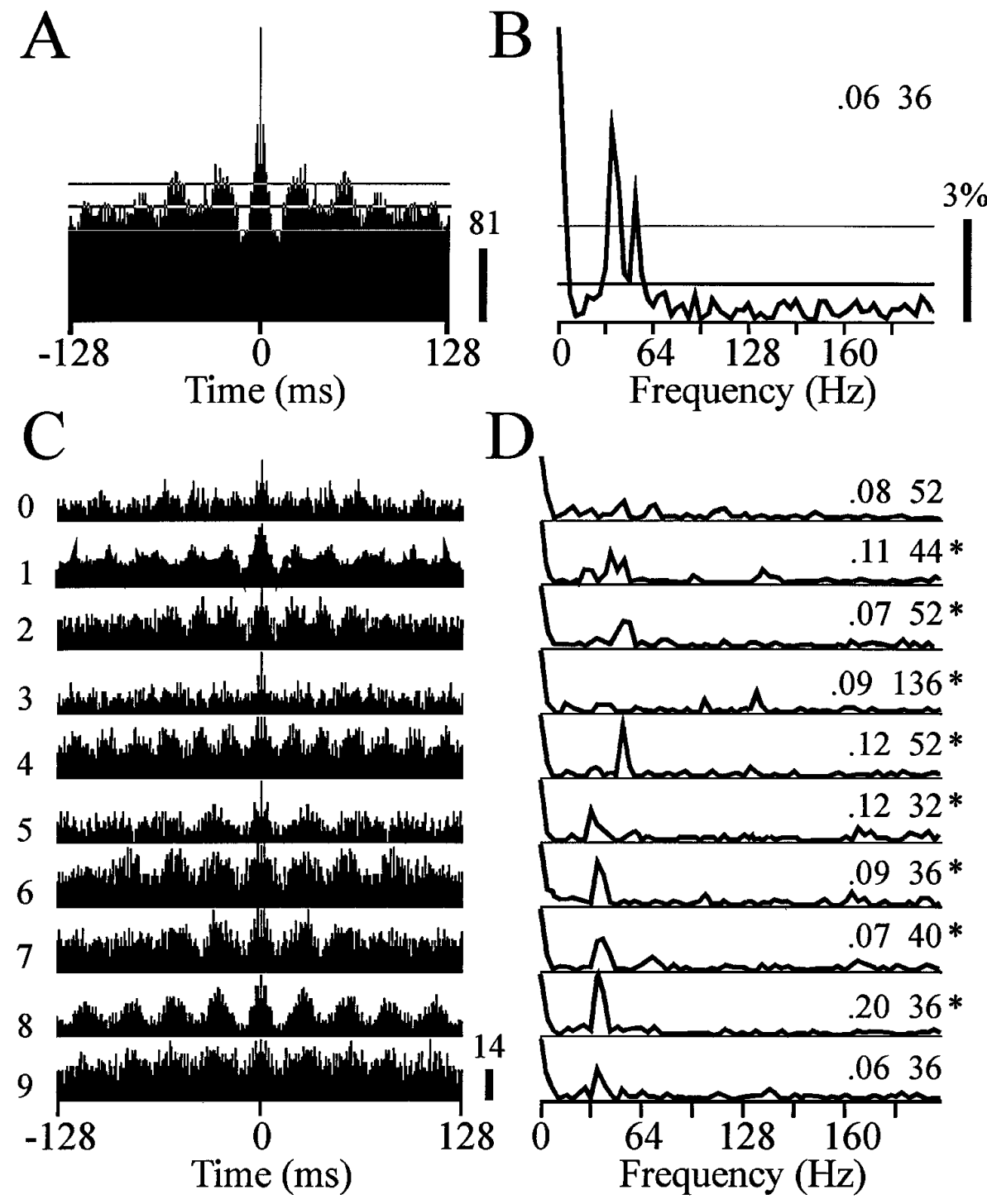

Figure 9. Example of a multiunit recording in which the cumulative autocorrelation histogram did not meet the criteria for significance (i.e., the peak/DC value is 0.06). $A$, Cumulative autocorrelation histogram. $B$, Corresponding power spectrum. The autocorrelation histograms and associated power spectra for each of the 10 trials recorded on this session are shown in $C$ and $D$, respectively. Eight of the 10 trials in this session met the criteria for significance. These are indicated by an asterisk to the right of the power spectra in $D$. the local temporal correlation among pairs or small groups of neurons. In some recordings, we were able to identify a pair of single units using principal components analysis, whereas in others, we extracted a single unit and a multiunit background signal using amplitude discrimination. We then performed cross-correlation analysis on these pairs of signals and applied the same statistical test for significance as that applied to the autocorrelation histograms. Figure 14 illustrates four examples of the raw data collected on single trials from single and/or multiunit recordings that were typical of the data base as a whole. Figure $14 A$ illustrates a single unit that exhibits a pattern of repetitive burst discharges that we refer to as "chattering" (Gray and McCormick, 1996). This pattern, which is also prevalent in area 17 and 18 of the anesthetized cat and area 17 of the alert monkey (Hubel and Wiesel, 1965; Gray et al., 1990, 1995b), consists of sequences of burst discharges that repeat at intervals of $15-50 \mathrm{msec}$ during the response to a visual stimulus. Within each burst, the firing rate ranges from roughly $300 \mathrm{~Hz}$ to as high as $800 \mathrm{~Hz}$. This pattern gives rises to the pronounced oscillations observed in the autocorrelation histograms and is often locally synchronized as shown in Figure $14, B$ and $D$. In Figure $14 B$, a chattering cell can be seen to fire with a slight but consistent phase lead over the lower amplitude multiunit background activity. In Figure $14 D$, there are several units firing in close temporal correlation. Such local synchronization does not, however, occur exclusively among cells that fire in repetitive bursts. Figure $14 C$ illustrates an example in which a single unit that displays no evidence of bursting or oscillation is tightly synchronized with the oscillatory multiunit activity recorded on the same electrode. This demonstrates that nonoscillating cells do participate among populations of neurons that display synchronous rhythmic firing.

Figure 15 illustrates an example of the local synchronization of oscillatory firing displayed by two cells recorded on the same electrode. These data were taken from the same recording shown in Figures 1-3 and demonstrate that oscillations in multiunit activity reflect the temporal synchronization of the underlying single units. The oscillations observed in each single unit are of the same frequency as the multiunit recording in Figure 3, and the two cells have an average time lag of $0 \mathrm{msec}$. Thus, the prevalence of significant rhythmicity in the discharges of multiunit recordings is indicative of a high degree of local temporal synchronization. 
A
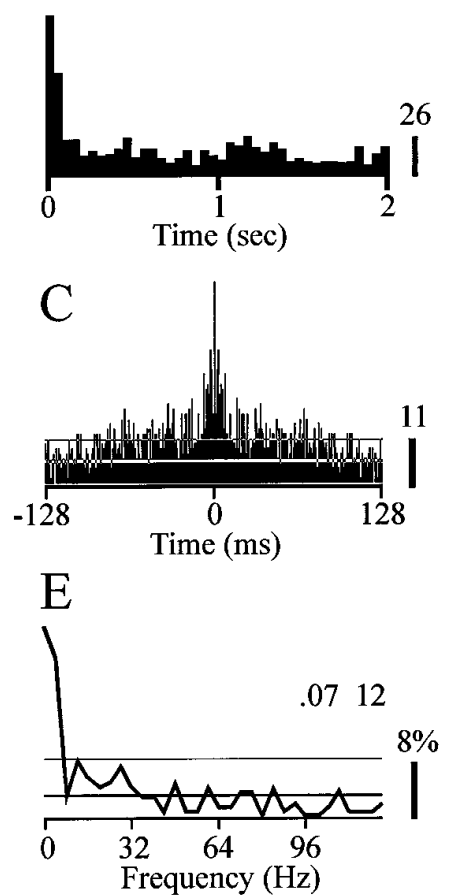

B
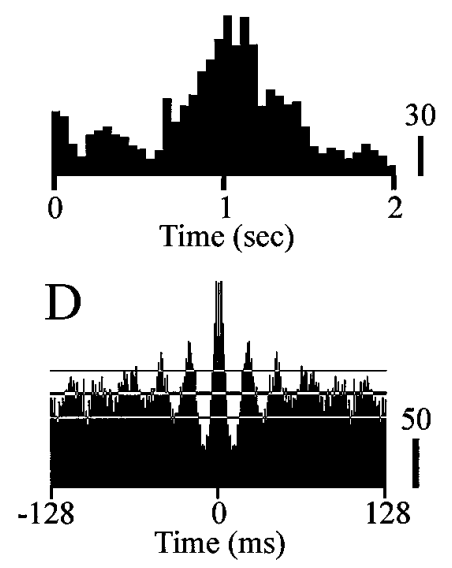

$\mathrm{F}$

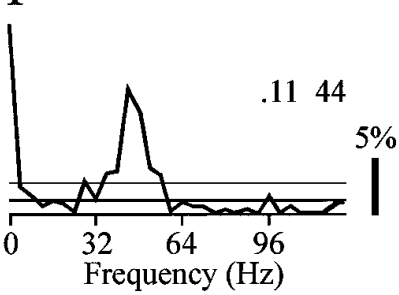

Figure 10. Oscillatory neuronal activity is present during visual stimulation but absent during periods of spontaneous firing. The left column illustrates the data obtained during fixation trials, whereas the right column illustrates the activity recorded during the presentation of an optimally oriented light bar. $A, B$, PSTHs. $C, D$, Autocorrelation histograms computed during the period of stimulus presentation. In $C$, this period ranged from 0.2 to 1.9 sec. $E, F$, Power spectra of the autocorrelation histograms shown in $C$ and $D$, respectively. Note the presence of a clear peak at $44 \mathrm{~Hz}$ in $F$ that is not present in $E$.

\section{DISCUSSION}

\section{Methodological considerations}

The occurrence and properties of stimulus-dependent oscillations in the visual cortex has been the subject of considerable debate. Much of this debate has focused on the probability of occurrence, the magnitude and statistical significance of neuronal oscillations. However, each of these parameters depends on the presence or absence of a sampling bias, the statistical test used to classify the data, and the criteria for what constitutes an oscillation. Concerning sampling bias, there are at least three possible sources of error, variation in the properties of oscillatory activity between animals, and inadequate or experimenter-biased sampling from individual animals. This study is subject to the first two of these sources of variation, which could account for the differences in the incidence and magnitude of oscillations between the alert and anesthetized states. However, we believe that the present data represent our best effort to avoid subjective biases in sampling the neuronal activity during data collection. Because of the constraints of recording from alert cats, we made a deliberate effort to collect every high quality recording we could obtain. This precluded us, for example, from searching for activity that gave more vigorous responses and were easier to map. In the anesthetized animals, we made electrode penetrations perpendicular to the cortical surface and sampled any multiunit or single unit activity that could be visually driven with a drifting light bar or square wave grating.

A $\quad$ B
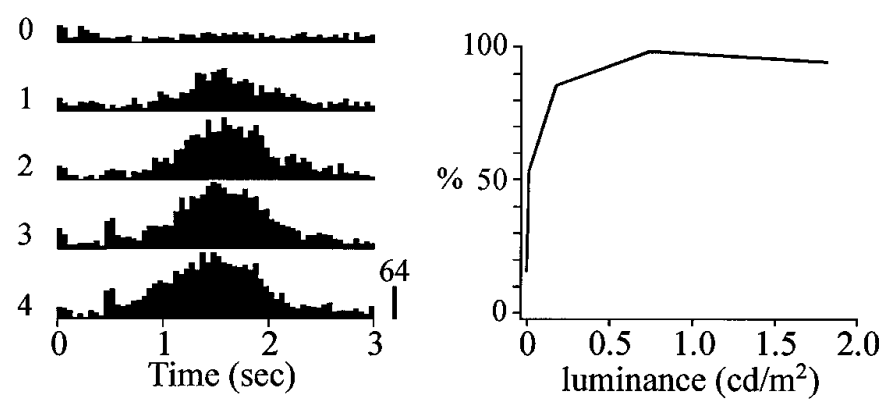

$\mathrm{C}$

0

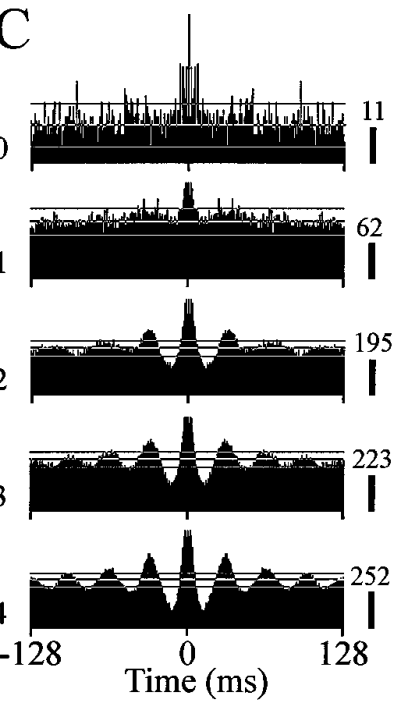

$\mathrm{D}$

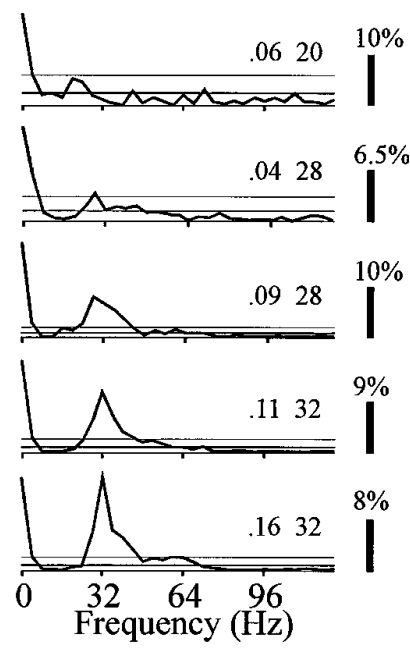

Figure 11. The magnitude of stimulus-evoked oscillatory firing increases with stimulus luminance. An optimally oriented light bar was passed over the receptive field of the recorded cells at five different levels of luminance. $A$, PSTHs computed from the spike trains recorded at each level of luminance. $B$, Tuning curve computed from the mean firing rate during the period of stimulus presentation. The plot is normalized to the maximum response. $C, D$, Autocorrelation histograms and corresponding power spectra computed from each of the five sets of spike trains, respectively.

The statistical tests used to measure and classify neuronal oscillations are another source of variability in results from different studies. The majority of investigations have used the autocorrelation histogram as the initial step in the quantification of rhythmicity (Gray and Singer, 1989; Gray et al., 1990; Engel et al., 1990; Ghose and Freeman, 1992; Young et al., 1992). The methods used to quantify the correlograms have differed, however. Our previous method of fitting a damped sine wave function to the autocorrelation histogram and then extracting the parameters of that function (Engel et al., 1990; König et al., 1994) have been criticized as being subject to error (Ghose and Freeman, 1992; Young et al., 1992). In an effort to address these criticisms and to make our results comparable to those of others, we applied the method of Ghose and Freeman (1992) of calculating the autocorrelation histogram, computing the power spectrum, and calculating a $\mathrm{S} / \mathrm{N}$ of the peak in the spectrum between $20-70 \mathrm{~Hz}$ relative to the average spectral values between $250-500 \mathrm{~Hz}$. Ghose and Freeman (1992) arbitrarily chose a $\mathrm{S} / \mathrm{N}$ value of 1.5 as indicative of significant periodicity in the $\mathrm{ACH}$. We applied this measure to our data and found that it classified a much greater percentage of 


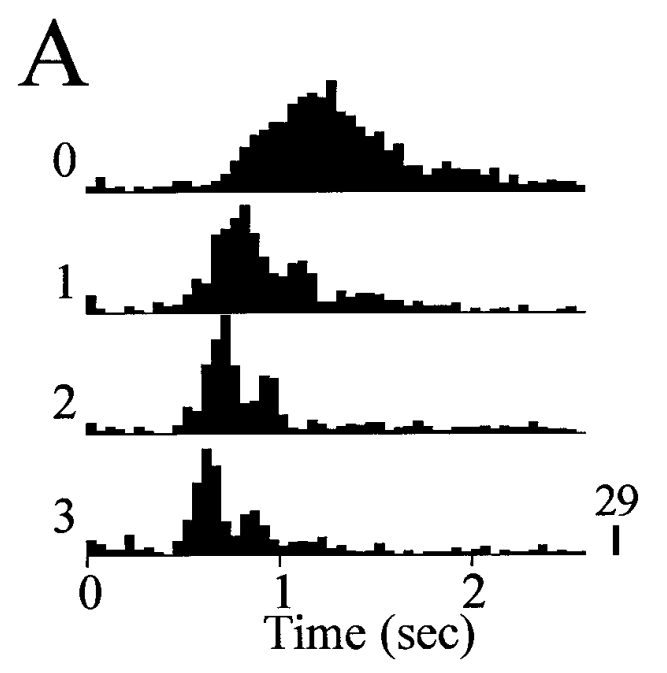

B
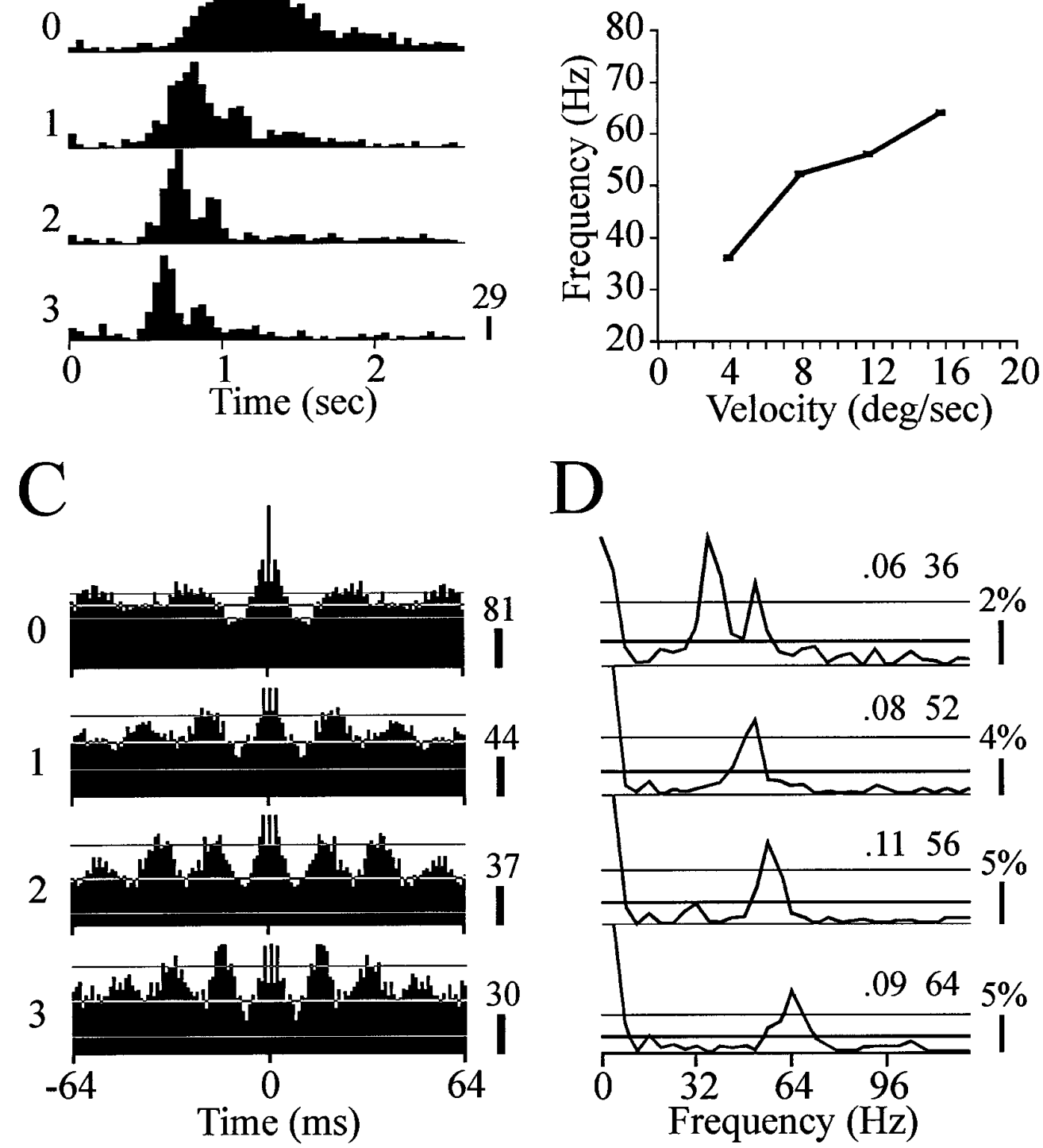

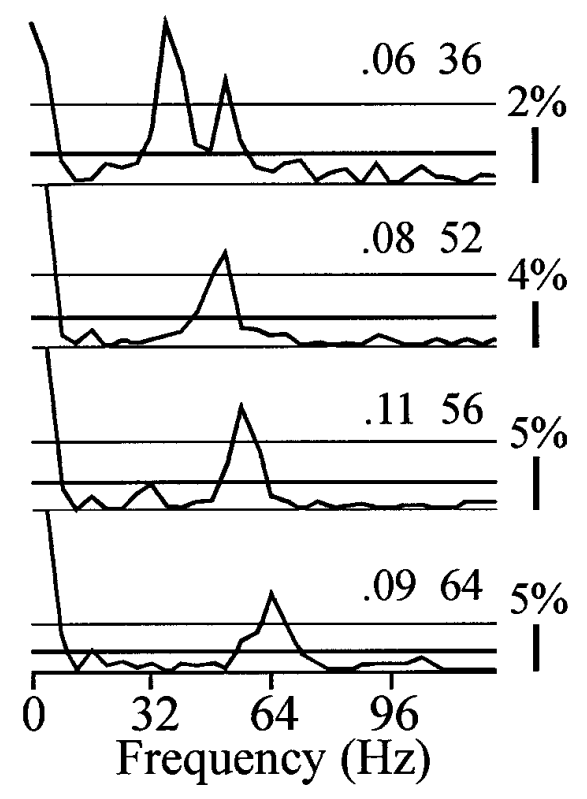

Figure 12. The frequency of oscillatory firing in the visual cortex of the alert cat increases with increasing stimulus velocity. Multiunit activity was recorded from a single electrode in area 18 , and moving light bars of high contrast were passed over the receptive field of the cell at four different velocities $\left(\sim 4,8,12\right.$, and $\left.16^{\circ} / \mathrm{sec}\right) . A$, PSTHs of the activity recorded in response to each of the 4 stimuli, velocity increasing from stimulus 0 to 3 . $B$, Tuning curve of the oscillation frequency computed from the power spectra shown in $D$. C, Autocorrelation histograms computed from the activity recorded during the response to each visual stimulus. $D$, Power spectra of the autocorrelation histograms shown in $C$. correlograms as oscillatory (Fig. 5) than obtained by previous estimates (Gray et al., 1990; Engel et al., 1990). In addition, we found that the $\mathrm{S} / \mathrm{N}$ measure did not take into account the total numbers of spikes used to compute the $\mathrm{ACH}$. To avoid this problem, we used a measure of the ratio of the peak in the spectrum to the DC component. To be classified as significant, the peak spectral values had to exceed the $99 \%$ confidence limit, computed from the $\mathrm{ACH}$ of an equivalent random spike train, and their peak/DC ratios had to be greater than $90 \%$ of the peak/DC values computed from the trial-shuffled histograms of the entire sample.

When we applied these relatively stringent criteria for statistical significance, we found a much lower incidence of oscillatory activity than revealed by the $\mathrm{S} / \mathrm{N}$ measure. This may account for the wide differences in results, and hence interpretations, reported by Ghose and Freeman (1992) and earlier studies (Gray and Singer, 1989; Gray et al., 1990; Engel et al., 1990). In support of this conclusion, we found that $\mathrm{S} / \mathrm{N}$ values of the recordings classified by the peak/DC measure as significant displayed a wide

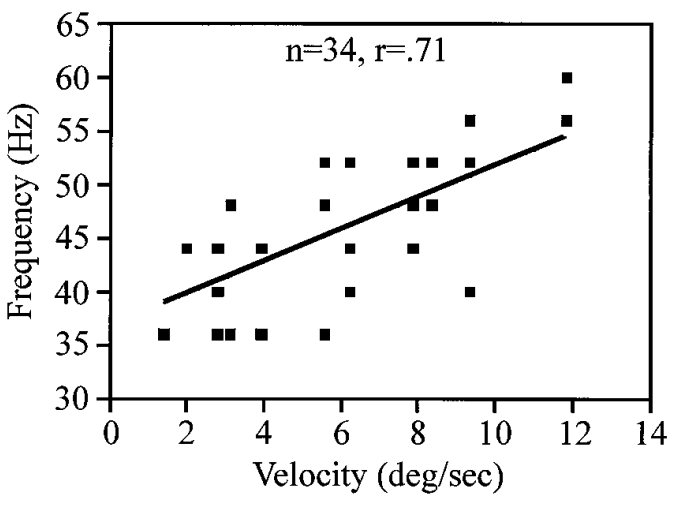

Figure 13. Scatter plot of the oscillation frequency, obtained from the peaks in the power spectra, versus the stimulus velocity for 11 multiunit recordings in area 17. Three to five different velocities were presented for each recording $(n=34)$. The linear correlation coefficient is $0.71(p \ll$ 0.0001 ) and the slope of the fitted line is $1.49 \mathrm{~Hz} / \mathrm{deg} / \mathrm{sec}$. 
A

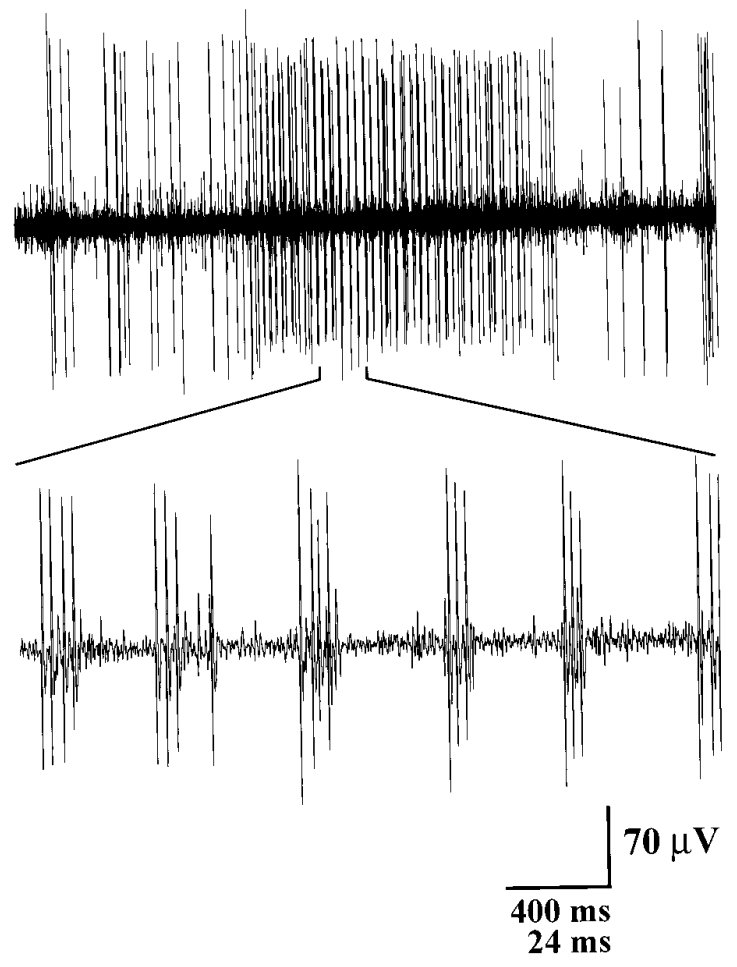

C
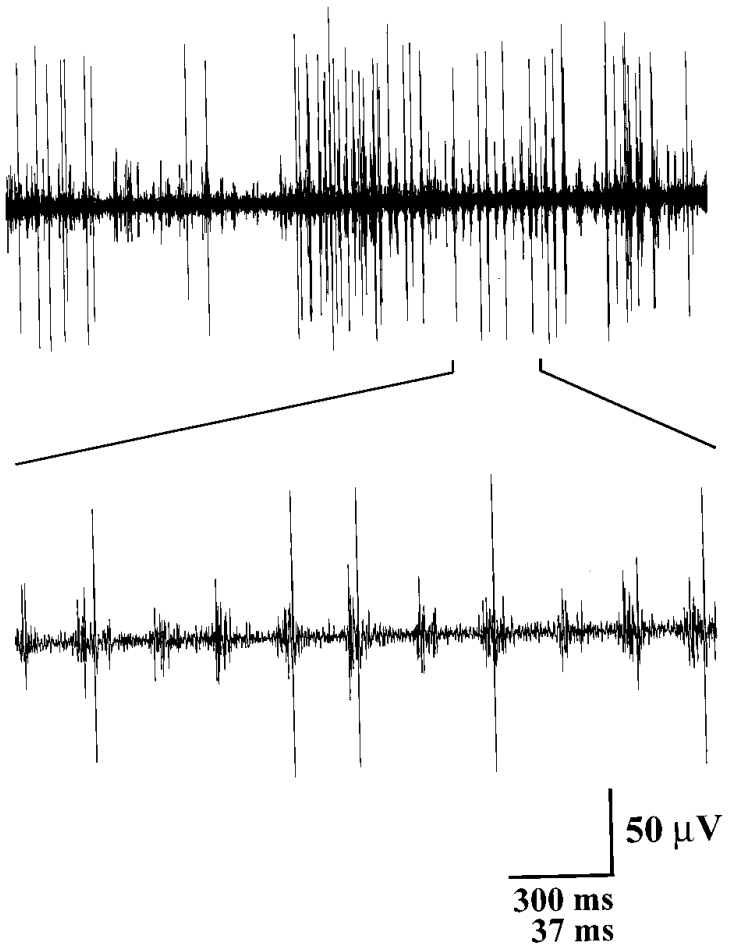

B
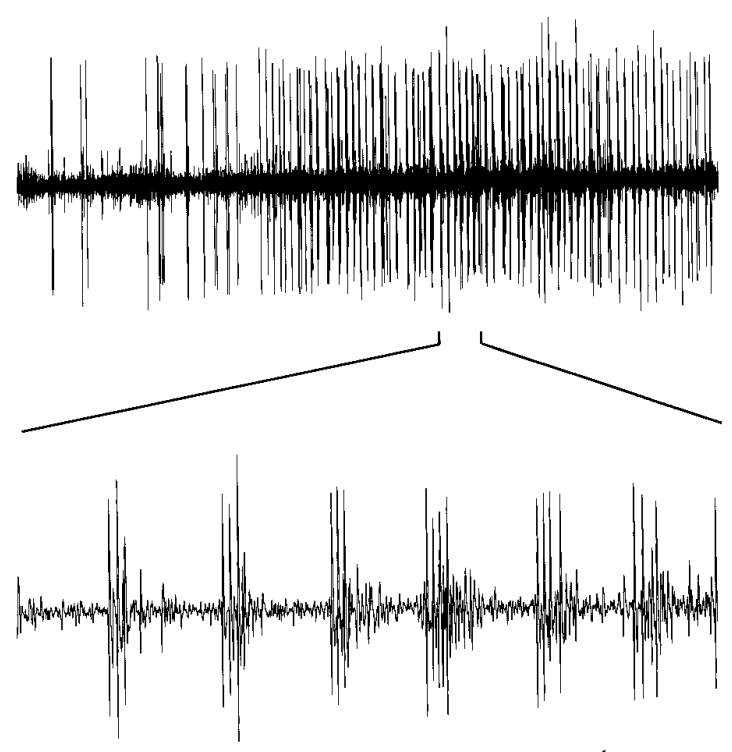

$30 \mu \mathbf{V}$

$22 \mathrm{~ms}$

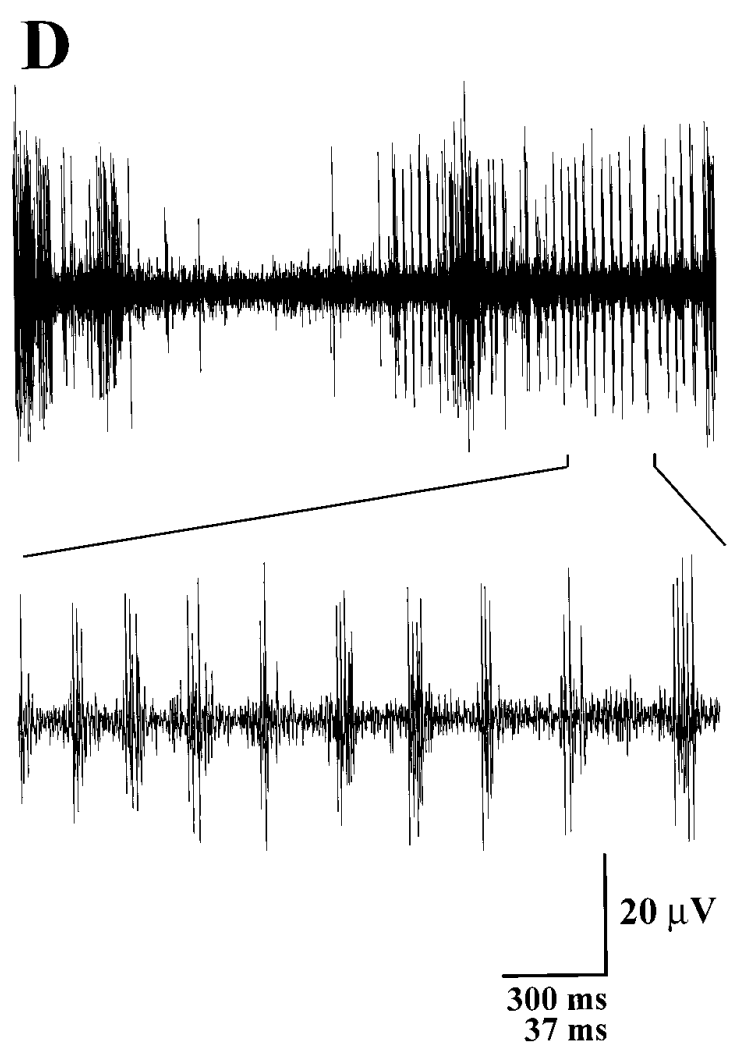

Figure 14. Four examples of oscillatory single and multiunit activity recorded on single trials in area 17 of the alert cat in response to an optimally oriented, drifting light bar. In each plot, the activity is displayed at a slow (top) and fast (bottom) time scale. The lines connecting the top and bottom plots indicate the epoch in the top plot that is being displayed in the bottom plot. $A$. Activity recorded from a single cell that exhibits a pattern of repetitive bursting during the visual response. $B$, A multiunit recording, consisting of a single isolated unit and lower amplitude background activity, illustrates that the repetitive burst discharges of the single unit are correlated with the multiunit activity. $C$, Another multiunit recording illustrating that a nonrhythmically firing single unit is synchronous with the local oscillatory multiunit activity. $D$, A further example of synchronous, rhythmic multiunit activity. 

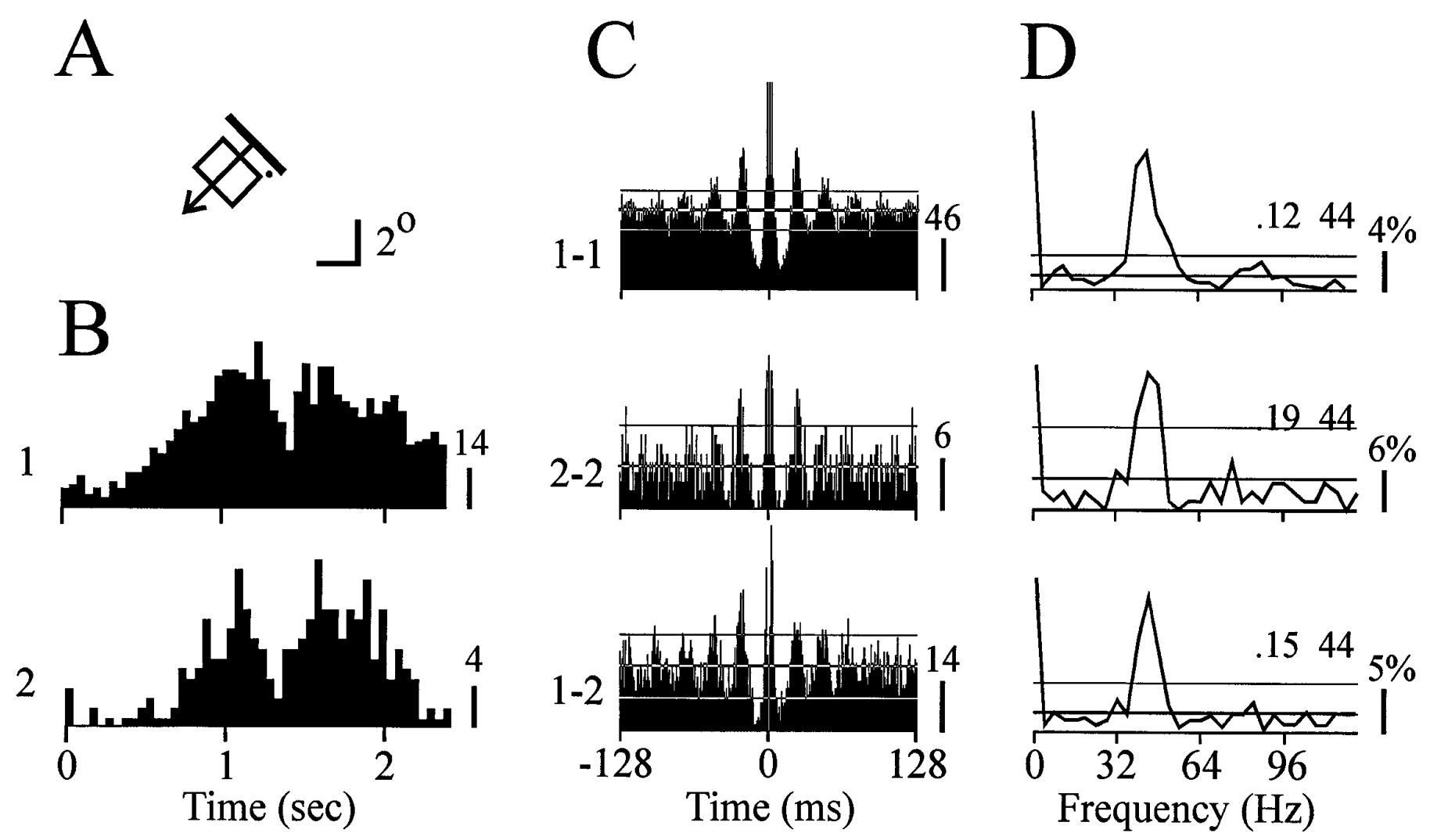

Figure 15. Synchronous rhythmic firing of two cells isolated from the multiunit recording shown in Figures 1-3. $A$, The receptive field of the multiunit activity and the stimulus used to activate the cells. The fixation spot is shown just to the right of the receptive field. $B$, PSTHs computed from the spike trains of the two cells $(1,2)$. C, Autocorrelation $(1-1,2-2)$ and cross-correlation (1-2) histograms computed from the two spike trains. The thick and thin lines represent the mean and $95 \%$ confidence limit computed from the same spike trains after shuffling the data by one stimulus period, respectively. $D$, Power spectra computed from the corresponding autocorrelation histograms. The numbers to the right of each graph are the peak/DC and the frequency of the peak value, respectively. The thick and thin lines represent the mean and $99 \%$ confidence limits computed from randomized spike trains having an equivalent number of spikes in the same interval of time, respectively.

range, and that many $\mathrm{S} / \mathrm{N}$ values greater than 1.5 did not meet our criteria for statistical significance. By utilizing an unbiased and stringent measure, it is also likely that the present results reflect a more accurate estimate of the incidence and magnitude of oscillatory activity than earlier studies (Eckhorn et al., 1988; Gray and Singer,1989; Gray et al., 1989, 1990; Engel et al., 1990).

It must be pointed out, however, that the cumulative $\mathrm{ACH}$ is not the best method for detecting and classifying neuronal oscillations in the visual cortex. Because neuronal oscillations can and often do exhibit wide variations in their frequency (Gray and Singer, 1989; Gray et al., 1992), summing these variations across trials in the cumulative $\mathrm{ACH}$ will tend to average out the periodicity (for example, see Fig. 9). This will lead to an underestimate of the incidence of rhythmic activity. Moreover, the magnitude of this underestimate is likely to increase as the number of trials collected increases and if spontaneous and stimulus-evoked activity are combined in the calculation of the correlation histograms. The traditional approach to correlation analysis has been to enhance the $\mathrm{S} / \mathrm{N}$ in the data by accumulating large numbers of spikes collected during periods of spontaneous and stimulusdriven activity (Perkel et al., 1967; Ts'o et al., 1986; Aiple and Krueger, 1988). The data presented here demonstrate that this practice is likely to mask rather than enhance the occurrence of rhythmicity in the spike trains.

Finally, because all visual stimulation was performed by computer, it is possible that refresh artifact (firing synchronized to the refresh frequency of the monitor) could account for some percentage of the oscillatory firing detected. Cells showing this behavior exhibit peaks in their correlation and shift predictor spectra having a frequency that is equal to, or a harmonic/subharmonic of, the refresh frequency. It was surprising that we did not find a single example of this effect in our data. It is, therefore, very unlikely that any of the significant spectral peaks observed in the correlograms reflect refresh artifact.

\section{Functional implications}

The results of this study demonstrate that synchronous gammaband activity in the visual cortex is not a phenomenon limited to anesthetized animals. It is robust and easily detectable on single trials in alert cats. Although problems of sampling bias preclude a firm conclusion regarding the differences between the alert and anesthetized data, the data presented here suggest that the incidence and magnitude of oscillatory activity is greater in the alert cats. These data are consistent with the recent findings that activation of the mesencephalic reticular formation (Munk et al., 1996) or the mesopontine cholinergic nuclei (Steriade et al., 1996) increase the probability and the magnitude of synchronous gamma-band activity in cat cortex. Thus, arousal seems to be an important variable influencing the occurrence and properties of gamma-band response synchronization.

The stimulus dependence of synchronous gamma-band activity, and its relative absence during spontaneous firing, indicate that 
neuronal oscillations are indeed related to visual processing, although in a manner which is not yet fully understood (Frien et al., 1994; Roelfsema et al., 1994; Livingstone, 1996; Kreiter and Singer, 1996). In their earlier study, Ghose and Freeman (1992) concluded that cortical gamma-band activity is not stimulus dependent and that oscillation strength is greatest at low firing rates. These results were complimented by the finding that a significant fraction of neurons in the LGN exhibit pronounced rhythmicity in the absence of visual stimuli, with a frequency distribution overlapping that of the cortical data (Laufer and Verzeano, 1967). In many of these thalamic neurons, the oscillations were suppressed or uninfluenced by visual stimuli. On the basis of these data, Ghose and Freeman (1992) proposed a model where cortical gamma-band activity is generated by the convergent input of independent but rhythmically firing LGN cells onto cortical neurons. Although this model seems to account for their data, it is inconsistent with at least two general findings presented in this and earlier studies: (1) gamma-band activity has been reported to be largely absent or greatly reduced in magnitude in the absence of visual stimulation (e.g., Figs. 2, 10, 11; Eckhorn et al., 1988; Gray and Singer,1989; Gray et al., 1990; Engel et al., 1990; Jagadeesh et al., 1992; Gray and McCormick, 1996); and (2) neuronal oscillations in the LGN of the anesthetized cat are stimulus dependent, both locally and globally synchronized (as long as they are driven by the same eye), and have a mean frequency near $80 \mathrm{~Hz}$ (Ito et al., 1994; Neuenschwander and Singer, 1996). These data, therefore, lead to the prediction that LGN oscillations should contribute to cortical gamma-band synchronization primarily during the response to visual stimuli. However, any model using this mechanism to generate cortical oscillations must account for the large difference in frequency distribution between the LGN and cortex.

Although the results presented here can shed little light on the underlying mechanisms of cortical gamma-band activity, one salient aspect is consistent with an alternative model for cortical gamma-band activity generation. When neuronal oscillations occur in the visual cortex, the cells contributing to these synchronous discharges often fire in a pattern of repetitive high frequency bursts (e.g., Fig. 14; Hubel and Wiesel, 1965; Gray et al., 1990, 1995b). These bursts recur at intervals of $15-50 \mathrm{msec}$ and can reach firing rates within a burst as high as $800 \mathrm{~Hz}$. Recently, using intracellular recording and staining in vivo, we have demonstrated that cells in striate cortex having these characteristics comprise a subpopulation of superficial pyramidal neurons (chattering cells) that, when injected with suprathreshold depolarizing current, exhibit intrinsic repetitive burst discharges at frequencies ranging from $20-70 \mathrm{~Hz}$ (Gray and McCormick, 1996). These cells also exhibit larger stimulus-evoked, gamma-band fluctuations in their membrane potential than any of the other cell classes. These findings suggest that a major component of visually evoked cortical gamma-band oscillations is influenced by the intrinsic membrane properties of chattering cells, and hence is a result of an intracortical mechanism. Cells that receive presynaptic input from chattering cells would, therefore, be expected to display membrane potential oscillations having properties consistent with a synaptic rather than an intrinsic mechanism (Jagadeesh et al., 1992). If chattering cells were synaptically connected to one another, the oscillatory activity in these cells would reflect both mechanisms. In view of the findings that oscillatory firing is a strong predictor of synchronization, these data suggest that chattering cells may play an important role in the generation of synchronous activity in visual cortex.

\section{REFERENCES}

Abeles M, Goldstein MH (1977) Multispike train analysis. Proc IEEE 65:762-773.

Aiple F, Krüger J (1988) Neuronal synchrony in monkey striate cortex: interocular signal flow and dependency on spike rates. Exp Brain Res 72:141-149.

Bair W, Koch C, Newsome W, Britten K (1994) Power spectrum analysis of bursting cells in area MT in the behaving monkey. J Neurosci 14:2870-2892.

Barlow HB, Blakemore C, Pettigrew JD (1967) The neural mechanism of binocular depth discrimination. J Physiol (Lond) 193:327-342.

Eckhorn R, Bauer R, Jordan W, Brosch M, Kruse W, Munk M, Reitboeck HJ (1988) Coherent oscillations: a mechanism of feature linking in the visual cortex? Biol Cybern 60:121-130.

Eckhorn R, Frien A, Bauer R, Woelbern T, Kehr H (1993) High frequency $60-90 \mathrm{~Hz}$ oscillations in primary visual cortex of awake monkey. NeuroReport 4:243-246.

Engel AK, König P, Gray CM, Singer W (1990) Stimulus-dependent neuronal oscillations in cat visual cortex: inter-columnar interaction as determined by cross-correlation analysis. Eur J Neurosci 2:588-606.

Engel AK, König P, Kreiter AK, Singer W (1991a) Interhemispheric sychronization of oscillatory responses in cat visual cortex. Science 252:1177-1179.

Engel AK, Kreiter AK, König P, Singer W (1991b) Synchronization of oscillatory neuronal responses between striate and extrastriate visual cortical areas of the cat. Proc Natl Acad Sci USA 88:6048-6052.

Friedman-Hill SR, Maldonado PE, Gray CM (1995) Short and longrange response synchronization in macaque striate cortex. Soc Neurosci Abstr 21:592.3.

Frien A, Eckhorn R, Bauer R, Woelbern T, Kehr H (1994) Stimulusspecific fast oscillations at zero phase between visual areas V1 and V2 of awake monkey. NeuroReport 5:2273-2277.

Ghose GM, Freeman RD (1992) Oscillatory discharge in the visual system: does it have a functional role? J Neurophysiol 68:1558-1574.

Gray CM, McCormick DA (1996) Chattering cells: superficial pyramidal neurons contributing to the generation of synchronous oscillations in visual cortex, Science 274:109-113.

Gray CM, Singer W (1987) Stimulus-specific neuronal oscillations in the cat visual cortex: a cortical functional unit. Soc Neurosci Abstr 13:404.3.

Gray CM, Singer W (1989) Stimulus-specific neuronal oscillations in orientation columns of cat visual cortex. Proc Natl Acad Sci USA 86:1698-1702.

Gray CM, Viana Di Prisco G (1993) Properties of stimulus-dependent rhythmic activity of visual cortical neurons in the alert cat. Soc Neurosci Abstr 19:359.8.

Gray CM, König P, Engel AK, Singer W (1989) Stimulus-specific neuronal oscillations in cat visual cortex exhibit inter-columnar synchronization which reflects global stimulus properties. Nature 338:334-337.

Gray CM, Engel AK, König P, Singer W (1990) Stimulus-dependent neuronal oscillations in cat visual cortex: receptive field properties and feature dependence. Eur J Neurosci 2:607-619.

Gray CM, Engel AK, König P, Singer W (1992) Synchronization of oscillatory neuronal responses in cat striate cortex: temporal properties. Vis Neurosci 8:337-347.

Gray CM, Maldonado PE, Wilson M, McNaughton B (1995a) Tetrodes markedly improve the reliability and yield of multiple single unit isolation from multiunit recordings in cat striate cortex. J Neurosci Methods 63:43-54.

Gray CM, Friedman-Hill SR, Maldonado PE (1995b) Stimulusdependent neuronal oscillations in areas V1 and V2 of the alert macaque monkey. Soc Neurosci Abstr 21:592.2.

Hubel DH, Wiesel TN (1965) Receptive fields and functional architecture in two nonstriate visual areas 18 and 19 of the cat. J Neurophysiol 28:229-289.

Ito H, Gray CM, Viana Di Prisco G (1994) Can oscillatory activity in the LGN account for the occurrence of synchronous oscillations in the visual cortex? Soc Neurosci Abstr 20:61.7.

Jagadeesh B, Gray CM, Ferster D (1992) Visually-evoked oscillations of membrane potential in neurons of cat striate cortex studied with in vivo whole cell patch recording. Science 257:552-554.

Judge SJ, Richmond BJ, Chu FC (1980) Implantation of magnetic search coils for measurement of eye position: an improved method. Vision Res 20:535-538.

König P (1994) A method for the quantification of synchrony and oscillatory properties of neuronal activity. J Neurosci Methods 54:31-37. 
König P, Engel AK, Roelfsema PR, Singer W (1995a) How precise is neuronal synchronization? Neural Comp 7:469-485.

König P, Engel AK, Singer W (1995b) The relation between oscillatory activity and long-range synchronization in cat visual cortex. Proc Natl Acad Sci USA 92:290-294.

Kreiter AK, Singer W (1992) Oscillatory neuronal responses in the visual cortex of the awake macaque monkey. Eur J Neurosci 4:369-375.

Kreiter AK, Singer W (1996) Stimulus-dependent synchronization of neuronal responses in the visual cortex of the awake macaque monkey. J Neurosci 16:2381-2396.

Laufer M, Verzeano M (1967) Periodic activity in the visual system of the cat. Vision Res 7:215-229.

Livingstone MS (1996) Oscillatory firing and interneuronal correlations in squirrel monkey striate cortex. J Neurophysiol 75:2467-2485.

Maldonado PE, Gray CM (1996) Heterogeneity in local distributions of orientation selective neurons in the cat primary visual cortex. Vis Neurosci 13:509-516.

Malpeli JG, Weyand TG, LaClair R (1992) A new method of mounting and directing chronically implanted microdrives. J Neurosci Methods 44:19-26.

Munk MHJ, Roelfsema PR, König P, Engel AK, Singer W (1996) Role of reticular activation in the modulation of intracortical synchronization. Science 272:271-274.

Neuenschwander S, Singer W (1996) Long-range synchronization of os- cillatory light responses in the cat retina and lateral geniculate nucleus. Nature 379:728-732.

Perkel DH, Gerstein GL, Moore GP (1967) Neuronal spike trains and stochastic point processes. II. Simultaneous spike trains. Biophys J 7:419-440.

Pettigrew JD, Cooper ML, Blasdel GG (1979) Improved use of tapetal reflection for eye-position monitoring. Assoc Res Vis Ophthal 18:490-495.

Press WH, Teukolsky SA, Vetterling WT, Flannery BP (1992) Numerical recipes in $\mathrm{C}$ : the art of scientific computing, Cambridge: Cambridge University.

Raether A, Gray CM, Singer W (1989) Intercolumnar interactions of oscillatory neuronal responses in the visual cortex of alert cats. Eur Neurosci Assoc Abstr 72.5.

Roelfsema PR, König P, Engel AK, Sireteanu R, Singer W (1994) Reduced synchronization in the visual cortex of cats with strabismic amblyopia. Eur J Neurosci 6:1645-1655.

Steriade M, Contreras D, Amzica F, Timofeev I (1996) Synchronization of fast $(30-40 \mathrm{~Hz})$ spontaneous oscillations in intrathalamic and thalamocortical networks. J Neurosci 16:2788-2808.

Ts'o D, Gilbert C, Wiesel TN (1986) Relationship between horizontal interactions and functional architecture in cat striate cortex as revealed by cross-correlation analysis. J Neurosci 6:1160-1170.

Young MP, Tanaka K, Yamane S (1992) On oscillating neuronal responses in the visual cortex of the monkey. J Neurophysiol 67:1464-1474. 